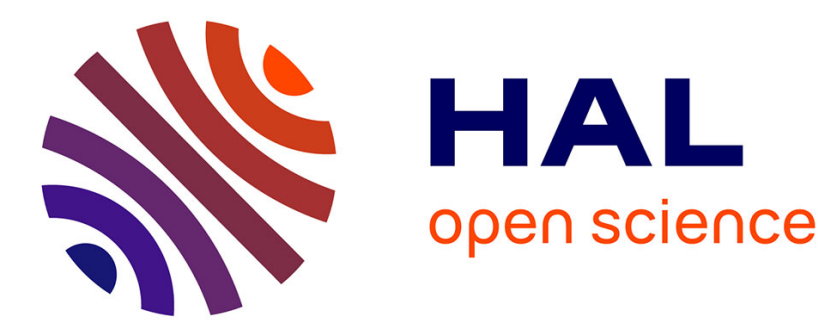

\title{
Modulateurs spatiaux bistables à cristal liquide et à adressage optique pour le calcul optique
}

Pierre Cambon, Jean-Louis de Bougrenet de La Tocnaye, Michael Killinger

\section{To cite this version:}

Pierre Cambon, Jean-Louis de Bougrenet de La Tocnaye, Michael Killinger. Modulateurs spatiaux bistables à cristal liquide et à adressage optique pour le calcul optique. Journal de Physique III, 1991, 1 (1), pp.45-67. 10.1051/jp3:1991107 . jpa-00248565

\section{HAL Id: jpa-00248565 https://hal.science/jpa-00248565}

Submitted on 1 Jan 1991

HAL is a multi-disciplinary open access archive for the deposit and dissemination of scientific research documents, whether they are published or not. The documents may come from teaching and research institutions in France or abroad, or from public or private research centers.
L'archive ouverte pluridisciplinaire HAL, est destinée au dépôt et à la diffusion de documents scientifiques de niveau recherche, publiés ou non, émanant des établissements d'enseignement et de recherche français ou étrangers, des laboratoires publics ou privés. 
Classification

Physics Abstracts

$42.30-42.80 \mathrm{~K}-$

\title{
Modulateurs spatiaux bistables à cristal liquide et à adressage optique pour le calcul optique
}

\author{
Pierre Cambon, Jean-Louis de .Bougrenet de la Tocnaye et .Michael Killinger \\ Groupe Optique et Systèmes de Communication, E.N.S.T. de Bretagne, B.P. 832, 29285 Brest \\ Cedex, France
}

(Reçu le $1^{\text {er }}$ mars 1990, révisé le 8 août 1990, accépté le 24 septembre 1990).

\begin{abstract}
Résumé. - On' présente les récents développements en matière de modulateurs spatiaux à cristal liquide à adressage optique pour le calcul optique. Le composant auquel on s'intéresse est conçu pour s'intégrer dans dés architectures faisant appel à des traitements impliquant un haut niveau de parallélisme et de connexionnisme. Les contraintes imposées à ce type de composant pour la réalisation d'architectures compactes de calcul optique sont précisées. Le principe de fonctionnement et l'expérimentation de l'adressage optique d'une valve à cristal liquide ferroélectrique bistable par deux photodiodes tête-bêche au silicium amorphe hydrogéné sont présentés et discutés.

Abstract. - Recent developments on optically addressed liquid crystal spatial light modulator are presented for their applications in digital optical computing. The aimed component will enable binary data handling capabilities of about 10 gigabit per second and per square centimeter. It is designed to be integrated in optical architectures involving a high level of parallelism and of interconnections. The component features, which are required for the implementation of compact optical computing architectures, are investigated. Operating principle and experimentation of the optical addressing of a ferroelectric liquid crystal bistable cell by using two positioned in a mirror image hydrogenated amorphous silicon photodiodes are presented an'd discussed.
\end{abstract}

\section{Introduction.}

La complexité croissante des tâches dévolues à un calculateur numérique (approches non algorithmiques, manipulation symbolique, ėc.) ainsi que l'augmentation du volume des traitements ont révélé l'inaptitude des architectures conventionnelles lors de l'implantation algorithmique de procédures supposant un haut niveau de parallélisme et surtout de connexionnisme ( $c f$. le numéro spécial d'Appl. Opt. [47]). Face à un tel enjeu et compte tenu de son aptitude naturelle aux traitements parallèles et connexionnistes, l'utilisation d'ondes électromagnétiques aux longueurs d'ondes optiques en espace libre offre une perspective intéressante dans le domaine du génie architectural. De ce point de vue, un des objectifs du calcul optique consiste à évaluer les performances et le réalisme de l'optique comme solution aux problèmes précédemment évoqués. 
Si les fonctions de calcul peuvent s'opérer par voie purement optique il n'en va pas de même des fonctions de contrôle ou d'entrées-sorties. Un des problèmes importants du calcul optique consiste à réaliser de façon satisfaisante ces fonctions indispensables sans trop pénaliser les temps de calcul. Une façon d'y arriver que l'on se propose d'illustrer ici est de transférer temporairement depuis son support optique vers un support matériel un grand volume d'informations pour lui appliquer globalement la fonction de contrôle par des moyens électriques simples.

La grandeur optique étant une grandeur spatiotemporelle, le problème d'un traitement peut être envisagé aussi bien d'un point de vue spatial que temporel. L'interaction ondematière fait intervenir une troisième composante qui est la durée d'interaction $\tau$ (temps de commutation, de mémorisation, etc.) largement supérieure au temps de propagation. Du point de vue du calcul optique, la quantité à considérer peut être la quantité d'informations véhiculée par le système, exprimée en bit/seconde, le nombre d'opérations effectuées par seconde (Mips) voire, dans le cas d'approche connexionniste, le nombre d'inférences logiques (Lips). C'est-à-dire des quantités basées sur le facteur $N^{2} / \tau$ voire $N^{3} / \tau$, où $N$ est le nombre de bits, d'opérations ou d'inférences traités pendant la durée $\tau$. Dans un tel contexte, il paraît raisonnable de chercher à agir en priorité sur le facteur $N$. Ce choix correspond en fait à privilégier les propriétés naturelles de l'optique visant au maximum de parallélisme et de connexionnisme [1].

Des travaux récents [12] permettent de penser que l'on pourra, dans un horizon pas trop lointain, réaliser des composants à l'état solide bistables ayant simultanément des temps de commutation très courts, des résolutions élevées et des sensibilités compatibles avec les diodes laser. De tels composants qui utilisent des empilements de couches extrêmement minces à base d'arséniure de gallium et présentent les caractéristiques requises définies précédemment permettront les réalisations compactes capables de traitements hors de portée des dispositifs purement électroniques. On pourra se reporter aux travaux récents portant sur la réalisation de circuits logiques basés soit sur des structures de type multi-puits quantiques [48] ou SEED (self electro-optic-effect device) [49] qui constituent une avancée importante dans le domaine de l'intégration de dispositifs de traitement numérique de l'information.

Nous proposons ici une alternative intéressante à ces solutions. Cette alternative consiste à privilégier l'utilisation de valves optiques à cristal liquide qui disposent d'un notable acquis technologique. Leurs temps de commutation certes modestes (quelque $10 \mu \mathrm{s}$ ) sont largement compensés par leur excellente résolution (quelque $10 \mu \mathrm{m}$ ) permettant d'espérer des flots de données binaires de l'ordre de la dizaine de gigabits par seconde et par centimètre carré comparables à ceux des architectures électroniques existantes.

Ces investigations sont encouragées d'une part par la recrudescence des travaux sur les cristaux liquides rapides dans la perspective du marché des écrans vidéo plats à mémoire intrinsèque $[14,15]$ et d'autre part par ceux sur les semi-conducteurs amorphes motivés par les applications photovoltaïques [16] et les écrans plats à matrice active [17].

\section{Traitement optique lié à l'emploi de modulateurs spatiaux.}

L'information est la valeur $G(x, y, t)$ d'une grandeur optique (intensité, polarisation, longueur d'onde, etc.). Dans le cas de l'utilisation de modulateurs spatiaux l'information est généralement codée sous forme binaire et l'on ne s'intéresse qu'à deux plages sans chevauchement de valeurs de $G(x, y)$ (conventionnellement appelées « 0 » et « $1 »)$. Pour créer une telle information binaire spatiale, on envoie un faisceau uniforme $(G(x, y)=C$ te $)$ sur un plan constitué d'une couche mince d'un matériau dont chaque point contient l'information binaire $\varphi(x, y)$ à transférer dans le faisceau sous forme de deux états physiques nettement distincts (indice de réfraction, coefficient d'absorption ou de réflexion, biréfrin- 
gence, etc.) capables d'agir sur la grandeur optique $G$. La résolution du matériau modulateur est le nombre maximum de paires d'états opposés que l'on peut aligner sur l'unité de longueur.

De ce point de vue les cristaux liquides se sont très tôt imposés comme matériaux modulateurs en raison de leur aptitude à être utilisés en couche mince, de leur grande résolution, de leur faculté à réaliser des fonctions logiques de calcul [3]. Les deux états physiques sont dans ce cas deux orientations différentes des molécules de cristal liquide créant une modulation spatiale de biréfringence agissant sur la polarisation du faisceau incident.

Le plan de matériau modulateur transmet ou réfléchit le faisceau incident uniforme en un faisceau dont la grandeur binaire $G(x, y)$ reproduit l'état binaire $\varphi(x, y)$. Cette opération constitue la lecture du modulateur spatial, elle est quasi instantanée. L'opération inverse est l'écriture: elle consiste à forcer dans le matériau modulateur les deux états physiques $\varphi(x, y)$ à l'aide d'un faisceau incident porteur d'information $G(x, y)$. Cette opération nécessite le maintien du faisceau d'écriture pendant un temps dit d'écriture qui est principalement fonction de l'inertie du matériau. L'énergie nécessaire pour commuter le matériau n'est que rarement disponible dans le faisceau d'écriture lui-même. En fait, celui-ci ne sert souvent qu'à désigner (adresser) par son paramètre $G(x, y)$ les points $(x, y)$ à commuter, l'énergie de commutation étant apportée par une source extérieure (dite source de pilotage).

Parmi les composants basés sur l'utilisation de modulateurs spatiaux à cristal liquide, la grandeur optique capable d'assurer un adressage est l'intensité $I(x, y)$. Par conséquent, même si les traitements optiques portent sur la grandeur $G(x, y)$ (par ex. la polarisation [2]), il sera nécessaire d'établir une correspondance entre ces deux grandeurs d'adressage et de calcul. Ce rôle est généralement dévolu aux composants appelés "valve optique " (light valve ou optically addressed spatial light modulator OASLM).

Ces composants reposent sur le principe suivant : on dépose sur le matériau modulateur une ou plusieurs couches dites de photoadressage, les zones éclairées de la couche de photoadressage dites «zones adressées " excitent par absorption des photons incidents des porteurs de charges libres qui sont acheminés par le champ électrique transversal créé par la source de pilotage vers le cristal liquide situé sous cette zone éclairée et qui va changer d'état. Evidemment, le cristal liquide situé sous des zones non adressées reste dans l'état initial. Il convient donc que l'ensemble du matériau modulateur soit dans un état initial connu avant d'envoyer le faisceau d'écriture. Autrement dit, toute écriture devra être précédée d'un effacement.

De nombreux composants de ce type [4-6] utilisant du cristal liquide nématique ont été réalisés dont le plus connu est celui de Hughes Aircraft [4]. Ces composants ont été employés avec succès dans de nombreuses architectures de traitement optique du signal [7-8]. Toutefois le cristal liquide nématique présente des inconvénients majeurs qui limitent son emploi dans des architectures de calcul optique. Il convient donc à ce stade d'analyser quelques aspects de ces architectures pour définir le composant convenable.

\section{Contraintes algorithmiques et architecturales.}

L'architecture la plus simple contient au moins deux OASLM (SLM1 et SLM2), un bloc de calcul optique bi ou tridimensionnel et deux sources pulsées (Fig. 2).

Le bloc intermédiaire (dit de calcul) opérant la mise en correspondance des deux modulateurs spatiaux peut être bidimensionnel (opération logique spatiale) ou tridimensionnel (algèbre matricielle, réseaux connexionnistes, etc.). Toutefois, comme on le verra par la suite, cette distinction n'affecte en rien la description algorithmique qui va être faite, elle est de nature purement architecturale (Fig. 1). 


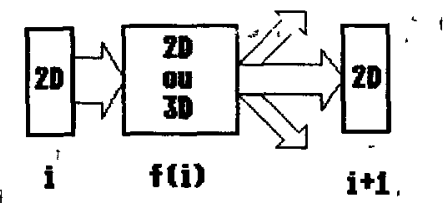

Fig. 1. - Synoptique d'une-instruction élémentaire.

[Elementary instruction synóptic.]

Le bloc de calcul peut être programmable en ce sens que l'ón peut changer sa configuration en un temps dit «de reprogrammation ». Toutefois, dès lors qu'il est configuré, lé calcul luimême s'effectue de façon purement photonique et donc en un temps corriespondant aúu temps de própagation de la lumière dans ce bloc que l'on peut négliger en regard des autres temps qui seront mis en jeu.

Dans l'état initial, figure 2a, le matériau modulàteur 1 est supposé conténir l'information à traiter sous forme physique $\varphi(x, y)$, le matériau modulateur 2 est supposé dans un état uniforme '(effacé). On supposera d'autre part que lés OASLM et le bloc de calcul transmettent l'information avec unè atténuation raisonnable.

Une première impulsion lumineuse projette l'information lue dans SLM1 sur les couches de photoadressage de SLM2 à travers le bloc de calcul. 'Cette impulsion' doit durer le temps d'écriture de SLM2. L'information de SLM1 doit rester stable durant ce tèmps. Donc un premier résultat 'est écrit dans SLM2 mais, dans une architecture itérative, il ne s'agit pas du résultat final.

La deuxième phase, figure $2 b$, consiste alors à effacer l'imáge initiale contenue dans SLM1. Ceci peut se faire de différentes façons, mais dure toujjours un temps $t_{\text {eff }}$ au moins égal au temps d'écriture en raison des phénómènes électro-optiques mis en jeu.

Il est "capital de noter que pendant ce temps, le $1^{\text {er }}$ résultat calculé doit rester mémorisé dans SLM2 alors que l'information qui a șervi à călculer ce résultat est en train de disparaître.

La troisième phase, figure $2 c$, est une phase analogue à la première, maìs généralèment sans calcul. C'est la phase dite «de rétroaction».

La quatrième phase, figure $2 \mathrm{~d}$, est identique à la deuxième phase avec permutation des plans 1 et 2 . Elle ramène le système dans l'état initial.

Les deux plans fonctionnent donc de façon rigoureusement symétrique et chacun passe successivement dans quatre phases sensiblement de même durée : écriture - mémoire - lecture - effacement. Les séquences des deux plans sont simplement décalées de 'deux phases l'une par rapport à l'autre. L'écriture et la lecture se font simultanément, mais dans des plans différents, de même pour la 'mémoire et l'effacement.

Dans ces conditions il est 'nécessaire que le composant puisse présenter deux états stables (état 0 et état 1). L'effacement consistera alors en un forçage uniforme à l'état « 0 » en un

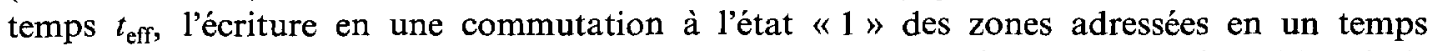
$t_{\text {ecr }}$ et le temps de mémoire $t_{\text {mem }}$ sera le temps pendant lequel un état « 1 » qui vient d'être écrit ne subit pas de dégradation suffisante pour qu'il risque d'être interprété comme un « 0 » à la lecture (immunité au bruit du système). Chaque plan doit donc mémoriser l'information pendant que l'autre s'efface puis pendant sa propre lecture (qui elle-même dure le temps d'écriture dans l'autre plan identique) ce qui impose :

$$
t_{\text {mem }} \geqslant t_{\text {eff }}+t_{\text {ecr }} \text {. }
$$

Une séquence de calcul complète dure alors:

$$
t_{\mathrm{cal}}=2 t_{\mathrm{eff}}+2 t_{\mathrm{ecr}} \text {. }
$$


(a)
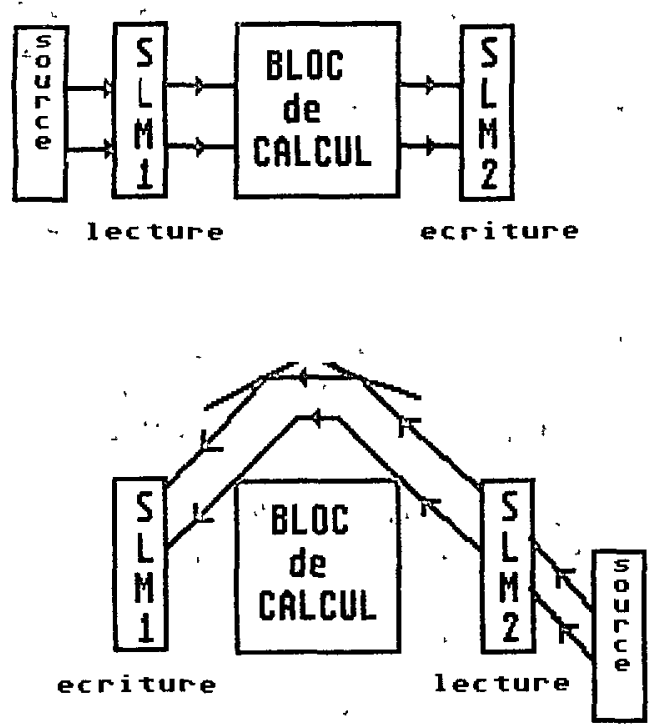

(c) (b)
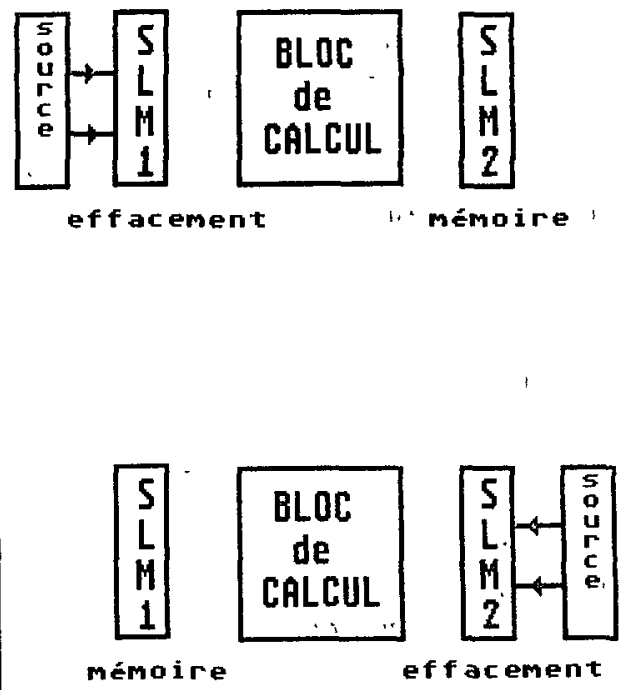

(d)

Fig. 2. - Développement algorithmique d'une instruction.

[Algorithmic development of one instruction.]

-Dans le cas particulier où les 2 états sont physiquement symétriques, les temps d'écriture et d'effacement deviennent égaux et on appelle temps de commutation $\left(t_{\text {com }}\right)$ la valeur commune, on a alors:

$$
t_{\mathrm{cal}}=4 t_{\mathrm{com}} \text {. }
$$

Dans la séquence de calcul représentée figure 2, le bloc de calcul n'est utilisé que dans la phase a, donc s'il est programmable, on dispose de $3 t_{\text {com }}$ pour le reprogrammer et donc-on peut y utiliser des composants 3 fois plus lents que pour les modulateurs spatiaux.

Le rôle fondamental des modulateurs spatiaux est d'assurer les-entrées-sorties - (non représentées en Fig. 2) et de régénérer l'information-à-chaque itération:- Eeúr rôle secondaire est de ménager la possibilité de reprogrammation du bloc dé calcul entre deux itérations.

La phase $b$ peut comporter un calcul en utilisant un deuxième bloc de calcul ou le même s'il est réversible. Ceci divise par 2 le nombre d'itérations nécessaires pour un traitement donné. A chaque séquence de calcul, on traite par unité de surface du modulateur spatial un nombre de bits égal au carré de la plus petite des résolutions qui entrent en jeu : celle du matériau modulateur, du photoadressage, de l'optique pendant un temps égal à $t_{\text {cal }}$ soit d'après (3) un flot de données binaires :

$$
F \text { bits } / \mathrm{s} / \mathrm{cm}^{2}=(\text { résolution })^{2} / 4 t_{\text {com }} \text {. }
$$

Les architectures reposant sur ce principe sont particulièrement intéressantes pour des procédures qui requièrent un traitement parallèle itératif de l'information comme cela est souvent le cas en traitement d'images [9], mais également pour des tâches d'un niveau de complexité supérieur telles que certaines approches d'intelligence artificielle [1]. 


\section{Contraintes imposées au composant.}

La contrainte fondamentale est celle imposée par l'inégalité (1) qui pénalise considérablement l'utilisation des cristaux liquides nématiques. Ces cristaux ne possèdent en fait qu'un seul état stable (l'état « 0 »). Dès la suppression du faisceau d'écriture, l'ensemble du matériau relaxe lentement vers cet état en un temps de plusieurs millisecondes $\left(t_{\text {eff }}\right)$ qui dépend de la viscosité du cristal. Les temps d'écriture de ces cristaux peuvent être relativement réduits en utilisant des tensions de pilotage de forte amplitude [10], mais la relation (2) imposera de toute façon des temps de calcul de l'ordre de $2 t_{\text {eff }}$ et donc des cadences de traitement ridiculement faibles ne permettant pas de mettre en évidence l'intérêt de ces architectures pour les traitements répétitifs pour lesquels elles sont conçues. Toutefois, pour des applications rapides, la relaxation lente du cristal liquide nématique pourrait constituer la mémorisation requise dans la mesure où l'on a les moyens de ramener le cristal dans son unique état stable rapidement par une action externe (par ex. magnétique).

Un important handicap de la plupart des réalisations de calcul optique est leur encombrement. Aussi convient-il d'emblée de rechercher des solutions intégrables. L'architecture proposée en est une illustration par l'utilisation d'un minimum de modulateurs spatiaux. Pour les composants, le même souci conduit à les concevoir d'emblée transmissifs plutôt que réflectifs. En effet, l'utilisation de composants transmissifs permet la mise en cascade de façon simple, tout particulièrement dans les architectures parallèles où elle peut consister à empiler des modulateurs. Ceci implique que les faisceaux d'écriture traversent les couches de photoadressage des plans lus. Compte tenu du rôle symétrique joué par ces plans, cette traversée des couches a lieu à une longueur d'onde où précisément elles doivent présenter une absorption notable pour être efficace à l'écriture. Comme on travaillera au bord de bandes d'absorption très larges par rapport à la gamme des longueurs d'ondes envisageables, il est illusoire de pouvoir résoudre ce problème en utilisant des longueurs d'onde différentes pour chacune des sources de la figure 2 et des couches de photoadressage différentes.

On utilisera donc une seule longueur d'onde et un seul type de couche de photoadressage. Le choix de la longueur d'onde constituera un compromis entre l'efficacité quantique et la transparence des couches de photoadressage.

Le composant logique est caractérisé par deux seuils d'intensité du faisceau d'écriture : un se uil bas en dessous duquel il n'y aura jamais commutation et un seuil haut au-dessus duquel il y aura tọiours commutation. Ces deux seuils ne peuvent être égaux et toute intensité située entre ces ân seüils-sera régénérée de façon aléatoire. Cette zone d'incertitude doit être minimisée car le bî̀o de calcul peut dégrader fortement le caractère binaire de l'information (bruits, diffraction, aberrations) que le composant est fơndamentalement chargé de régénérer.

En ce qui concerne la distinction possible entre architecture connexionniste ou simplement parallèle, on peut remarquer qu'une architecture parallèle conduit à faire le choix d'un composant transmissif. Une solution connexionniste, basée notamment sur l'utilisation de réseaux holographiques, telle que décrite par exemple en [11], autorise les deux choix (en transmission ou réflexion). La seule contrainte, qui résulte dans ce cas au niveau du composant, concerne la nécessité de pouvoir tolérer un angle de vue important $\left( \pm 30^{\circ}\right)$. Ceci est le cas notamment de la solution que nous allons proposer maintenant [35].

\section{Les cristaux liquides ferroélectriques.}

Parmi les nombreuses phases de cristal liquide, on observe à température plus basse que pour la phase nématique une phase dite «smectique » où les molécules de forme allongée (de vecteur directeur n) s'organisent en couches équidistantes et parallèles (Fig. 3). Dans la phase 


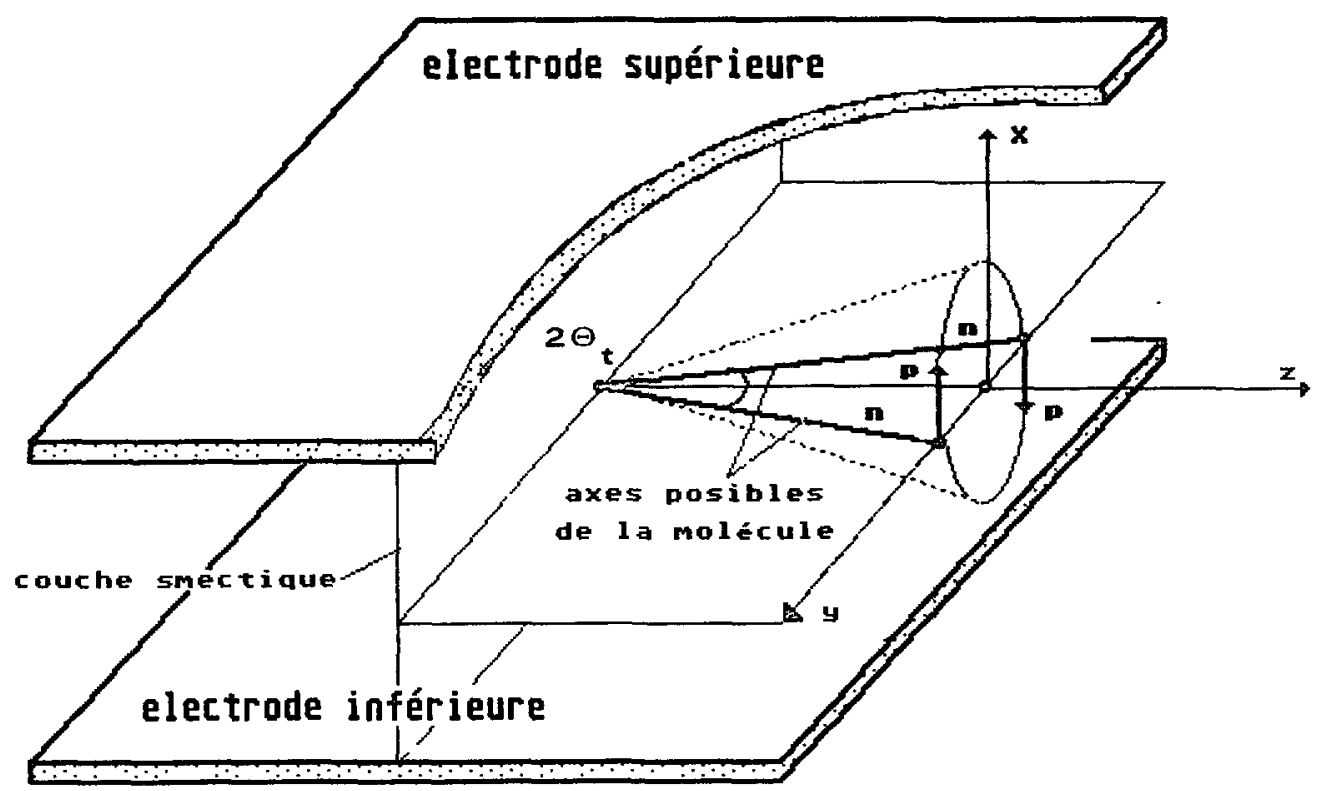

Fig. 3. - Configuration simplifiée d'une cellule ferroélectrique à cristal liquide $\left(\mathrm{SmC}^{+}\right)$.

[Configuration of ferroelectric smectic $\mathrm{C}^{*}$ liquid crystal cell.]

smectique $C$, sur une même couche, les molécules sont parallèles et inclinées d'un angle $\theta_{\mathrm{t}}$ appelé angle de « tilt » sur la normale aux couches. Si de plus les molécules sont chirales (i.e. non superposables à leur image dans un miroir), elles s'alignent de façon à entraîner l'apparition d'une polarisation permanente noté $\mathbf{P}_{\mathrm{s}}$ (polarisation spontanée) perpendiculaire au vecteur $\mathbf{n}$ et parallèle aux couches. Les interactions intermoléculaires couche à couche entraînent naturellement une rotation $\mathrm{du}$ vecteur directeur (et donc de la polarisation spontanée) dont l'extrémité décrit une hélice tout en restant sur le cône d'ouverture $2 \theta_{t}$ appelé cône smectique. Un cristal liquide présentant une telle structure est noté $\mathrm{C}^{*}$ (ou $\mathrm{SmC}^{*}$ ). Pour pouvoir observer une polarisation spontanée macroscopique et donc rendre le matériau ferroélectrique, il faut dérouler l'hélice. Ces propriétés ferroélectriques des cristaux liquides $C^{*}$ ont été mises en évidence en 75 par Meyer (Orsay) [18]. Il est à noter que les cristaux liquides $\mathrm{SmC}^{*}$ sont des mélanges incorporant des dopants chiraux dans des mélanges non chiraux. Dans ces dopants, on fait intervenir des composants dont les hélices « tournent " en sens inverse si bien qu'il est possible de "dérouler» chimiquement l'hélice du mélange. Cette opération peut, de plus, renforcer la valeur de la polarisation spontanée.

La première utilisation comme obturateur optique des cristaux liquides $\mathrm{SmC}^{*}$ a été proposée par Clark et Lagerwall (Fig. 3). Une couche de cristal liquide d'épaisseur (de l'ordre. du micron) nettement inférieure au pas de l'hélice est confinée entre deux lames de verre perpendiculaires aux couches. Dans cette structure dite SSFLC (cellule ferroélectrique stabilisée par les surfaces), le vecteur polarisation spontanée $\mathbf{P}_{\mathrm{s}}$ d'amplitude $P_{\mathrm{s}}$ ne peut prendre que deux positions dont la projection sur l'axe perpendiculaire aux surfaces est $+P_{\mathrm{s}}$ (up) ou $-\boldsymbol{P}_{\mathrm{s}}$ (down). Ceci conduit à l'observation de domaines stables où la polarisation spontanée est uniforme à l'état up ou down (analogues aux bulles magnétiques). Ces domaines sont séparés par des murs très fins dont l'épaisseur est de l'ordre de celle de la cellule. Le vecteur directeur de la molécule (i.e. l'axe optique de la cellule) dans un domaine 
up ou down est parallèle aux lames de verre, mais son angle par rapport à la normale aux couches vaut $-\theta_{\mathrm{t}}$ dans un domaine up et $+\theta_{\mathrm{t}}$ dans un domaine down par exemple. Le passage d'un domaine dans un autre 's'accompagne donc d'une rotation de l'axe optique de $2 \theta_{t}$. Pour un choix judicieux de ce paramètre, la cellule se comporte alors comme une lame de phase $\lambda / 2$.

Lorsque la cellule est disposée entre deux polariseurs croisés orientés suivant ses axes propres, un de ces états est transmissif tandis que l'autre est absorbant. Lorsqu'on applique un champ électrique d'amplitude $E$ perpendiculairement aux lames, les molécules sont soumises essentiellement à un couple qùi tend à orienter les molécules de façon à ce que $\mathbf{P}$ et E soient parallèles et de même sens. On peut donc faire commuter l'état d'une cellule SSFLC en lui appliquant un champ de sens adéquat. Les temps de commutation son' beaucoup plus courts que pour les nématiques car le champ électrique interagit dans les cristaux liquides ferroélectriques avec la polarisation spontanée qui est beaucoup plus élevée que la polarisation induite $\varepsilon E$ qui intervient seule dans le cas des cristaux non ferroélectriques. Le temps de commutation est limité par la viscosité rotationnelle $\gamma$ du cristal [19] :

$$
t_{\text {com }} \equiv \gamma / \mathbf{P}_{\mathrm{s}} \cdot \mathbf{E} \text {. }
$$

Des temps de commutation inférieurs à la microseconde entre deux états 'stables ont été observés sous des tensions de $40 \mathrm{~V}$ et à une température de $68{ }^{\circ} \mathrm{C}$ [19]. De tels matériaux conviennent donc parfaitement pour des architectures de calcul optique. Ils possèdent en outre une capacité de mémorisation de longue durée qui permet de les utiliser dans des architectures plus complexes que celles de la figure 1 où des résultats intermédiaires doivent être mémorisés au cours de séquences complexes de calcul. Ce point est fondamental car il permet de contourner une des principales limitations actuelles des architectures numériques liée à la séparation des tâches processeur-mémoire.

Toutefois, en raison des difficultés théoriques et technologiques spécifiques de ces matériaux, ils n'ont été employés que très récemment dans des architectures de 'calcul optique. En effet, le modèle SSFLC décrit plus haut n'est qu'une approche très simplifiée de
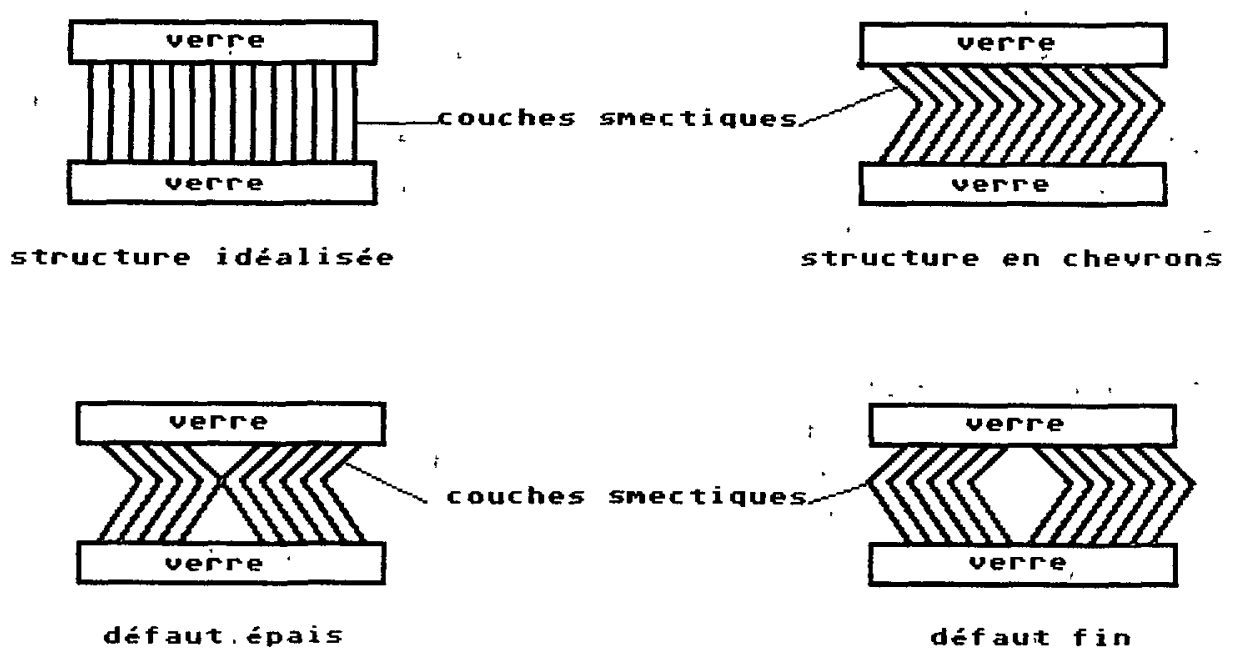

Fig. 4. - Défauts «zig-zag " d'une cellule réelle.

[Zig-zag defects in SSFLC.] 
la réalité observée. En 87, par des analyses aux rayons $X$ à haute définition [20], il a été montré que les couches smectiques n'étaient,pas perpendiculaires aux lames mais s'inclinaient pour former des chevrons. D'autre part, les positions des vecteurs directeurs des molécules d'une même couche en chevron sur le cône smectique varient suivant la distance aux surfaces et en un nombre fini de configurations clairement répertoriées que l'on appelle la structure des états et qui est d'autant plus complexe que l'épaisseur du cristal est grande. L'arrangement réel du cristal liquide et a fortiori la modification de ces arrangements par application d'un champ extérieur sont loin d'être à ce jour parfaitement connus. La réponse optique qui en résulte est complexe et exclut une logique binaire fondée sur un codage de polarisation. Enfin, les observations et les interprétations dépendent fortement de la technologie de réalisation de la cellule ainsi que de la composition des mélanges utilisés pour obtenir le cristal liquide. Les expériences que nous avons effectuées avec des cellules SSFLC fabriquées pär le LETI [21-22] permettent toutefois de mieux situer les problèmes.

\section{Expérimentation sur une cellule mince.}

Les oscillogrammes de la figure 5 ont été obtenus avec des cellules SSFLC réalisées 'par le LETI d'une épaisseur de $1,5 \mu \mathrm{m}$ de cristal liquide $\mathrm{SmC}^{*}$ (Felix 001 de chez Hoechst) présentant à $20^{\circ} \mathrm{C}$ une polarisation spontanée de $7,5 \mathrm{nC} / \mathrm{cm}^{2}$, une viscosité rotationnelle de $64 \mathrm{mPa} . \mathrm{S}$, un angle de tilt de $32^{\circ}$. Le champ électrique longitudinal de commutation est obtenu par application de la. tension de pilotage entre deux électrodes transparentes (ITO) séparées des couches d'orientation (polymère frotté) en contact avec le cristal liquide par unè couche intermédiaire dé silice (épaisseur $600 \AA$ ). On applique des tensions de faible amplitude $( \pm 3 \mathrm{~V}$, Fig. 5a) compatibles avec la configuration de photoadressage par photodiode en silicium amorphe-hydrogéné qui sera proposée par la suite. Cette tension à valeur moyenne nulle est constituée d'impulsions rectangulaires positives et négatives. On éclaire la cellule à $633 \mathrm{~nm}$ (laser $\mathrm{He}-\mathrm{Ne}$ ) et on forme l'image d'un pixel de $300 \mu \mathrm{m} \times$ $300 \mu \mathrm{m}$ sur un détecteur rapide (photodiode à avalanche au silicium), deux polariseurs croisés sont interposés avant et après la cellule. Sur la photo figure $5 \mathrm{c}$, les polariseurs croisés sont réglés par rapport à la cellule pour'obtenir des 'réponses optiques identiques sur des impulsions de pilotage positives et négatives. Par rapport à cette position (que l'on appellera provisoirement de référence) une inclinaison des polariseurs (de $7,5^{\circ}$ dans un sens donne la photo Fig. $5 \mathrm{~b}$ et de $7,5^{\circ}$ dans l'autre sens la Fig. $5 \mathrm{~d}$ sur lesquelles on observe 4 états stabilisés). $\mathrm{A}+$ se stabilise après environ $1 \mathrm{~ms}$ d'application de la tension positive, cet état relaxe en $1 \mathrm{~ms}$ également vers l'état $\mathbf{M}+$ dès que la tension appliquée redevient nulle. Symétriquement, l'état A- se stabilise en $\mathbf{l} \mathrm{ms}$ sous une tension négative puis relaxe vers $\mathbf{M}-$ à l'annulation de la tension. Sur la figure $5 \mathrm{~b}$, les polariseurs croisés sont réglés pour que l'état $\mathbf{M}+$ corresponde à une intensité transmise minimum.

Lorsque l'on éteint à un instant aléatoire la source de tension de pilotage (court-circuit) de la cellule, elle se stabilise, pour une orientation de polariseurs donnée, dans l'état $\mathbf{M}+$ ou bien M- de façon équiprobable. Ces états peuvent rester inchangés indéfiniment (plusieurs mois). Il s'agit des 2 états stables (dits aussi : relaxés ou mémorisés) du matériau modulateur selon la terminologie, définie plus haut. Lorsque la cellule est abandonnée court-circuitée dans l'état $\mathbf{M}-$ (respectivement $\mathbf{M}+$ ), on observe qu'elle se comporte comme une lame biréfringente dont un axe est incliné à $-7,5^{\circ}$ (respectivement $+7,5^{\circ}$ ) par rapport à la position de référence. On peut parler d'axes optiques mémorisés. Ces axes font entre eux un angle de $15^{\circ}$ et sont symétriques par rapport à la position de référence.

Les états $\mathrm{A}+$ et $\mathrm{A}-$ peuvent également être éteints pour des positions des polariseurs symétriques par rapport à la référence, mais leur angle (de l'ordre de $45^{\circ}$ ) est plus grand que celui des états mémorisés. Ces deux états sont appelés états adressés ou commutés. Par raison 

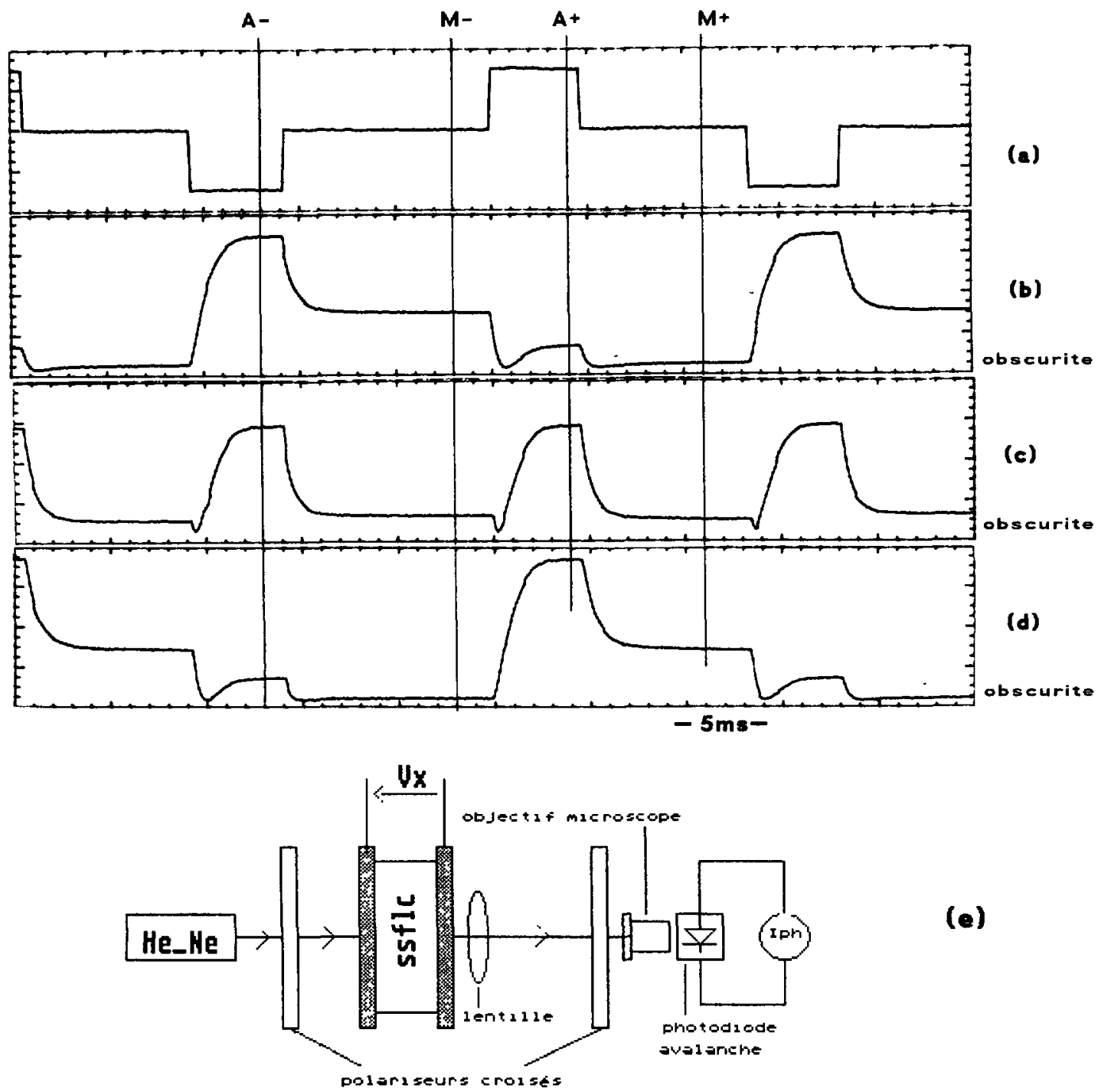

(e)

Fig. 5. - Intensité transmise par une cellule SSFLC, entre polariseurs croisés, pour diverses orientations, sous faible tension de pilotage : a) impulsions symétriques de tension $\left(V_{\mathrm{x}}\right)$ appliquées aux bornes de la cellule $( \pm 3$ volts) ; b) orientation au maximum d'absorption dans l'état mémorisé $\mathrm{M}^{+}$; c) orientation fournissant des réponses optiques symétriques, position de référence ; d) orientation au maximum d'absorption dans l'état mémorisé $\mathbf{M}^{-}$; e) montage utilisé.

[Transmitted intensity response of a SSFLC, between two crossed polarizers, driven by low voltage pulses versus the orientation of the cell : a) symmetrical voltage driving pulses $V_{\mathrm{x}}( \pm 3$ volts); b) orientation setting for maximum absorption in a $\mathbf{M}^{+}$memorized state ; c) orientation setting for symmetrical optical response (reference); d) orientation setting for maximum absorption in a $\mathbf{M}^{-}$ memorized state; e) experimental set-up used.]

de symétrie, les deux états adressés et les deux états mémorisés correspondent physiquement à des configurations symétriques des molécules par rapport à la normale aux couches smectiques. Or celle-ci est imposée à la construction par la direction de frottage du polymère 
d'orientation. La position de référence du polariseur conduisant à la réponse optique symétrique de la photo figure $5 \mathrm{~d}$ correspond donc physiquement à la direction de frottage de la cellule.

Dans l'orientation de la figure $5 \mathrm{~b}$, la lumière émergente de la cellule n'est polarisée rectilignement que pour l'état $\mathbf{M}+$ qui peut donc quasiment être éteint par l'analyseur, mais l'état $M$ - est de polarisation elliptique. La lecture d'une information mémorisée dans la cellule ne fournit donc pas un faisceau codé suivant deux états perpendiculaires dè polarisation rectiligne comme ceci est requis dans certaines architectures, notamment pour permettre l'inversion logique par simple interposition d'une lame $\lambda / 2$. On peut noter toutefois que la cellule dans l'état adressé peut jouer le rôle d'une lame $\lambda / 2$ programmable puisque l'angle entre les 2 états $\mathrm{A}+$ et $\mathrm{A}$ - est voisin de $45^{\circ}$. La rotation de $15^{\circ}$ du polariseur qui intervient entre les figures $5 \mathrm{~b}$ et $5 \mathrm{~d}$ opère une permutation des réponses des états + et (A et $\mathrm{M}$ ) et donc une inversion logique.

Ces observations montrent que le comportement réel d'une cellule SSFLC s'éloigne notablement du modèle simplifié de Clark et Lagerwall. La théorie montre que l'angle entre les deux états mémorisés est toujours inférieur à celui des états commutés lui-même inférieur à l'angle du cône smectique comme on l'observe.

\section{Perspectives d'optimisation des couches modulatrices à cristaux liquides ferroélectriques.}

Les travaux d'optimisation des cristaux liquides ferroélectriques sont poursuivis depuis quelques années dans la perspective de réalisation d'écrans plats [23-24]. Cette finalité implique des contraintes qui sont sensiblement éloignées de celles qu'impose une perspective de calcul optique qui commence à peine à être envisagée. Nous examinerons ici l'optimisation des paramètres de contraste des états mémorisés, le temps de commutation et la résolution dans le cadre défini par les contraintes indiquées plus haut.

- Le contraste des états mémorisés, qui constitue une mesure du caractère bistable du composant, peut être amélioré de 3 façons différentes.

La première consiste à diminuer l'épaisseur de la cellule $(<1 \mu \mathrm{m})$; ceci étend l'influence des forces d'orientation de surface vers le centre de la cellule qui tend à se comporter comme le modèle simplifié avec notamment une augmentation de l'angle entre les états mémorisés vers l'angle du cône smectique. Le contrôle d'une telle finesse a été obtenu par le LETI à $\pm 0,1 \mu \mathrm{m}$ à l'aide de plots de résine photolithographiés régulièrement espacés $(50 \times 50 \mu \mathrm{m}$ espacés de $300 \mu \mathrm{m}$ ) [21]. Ce contrôle obtenu pour des surfaces de $100 \mathrm{~cm}^{2}$ peut a fortiori être obtenu pour des surfaces de $10 \mathrm{~cm}^{2}$ que l'on envisage en calcul optique, mais les plots imposent une trame de zones inactives (noires entre polariseurs croisés) qui se superpose à l'information traitée. Se pose alors le problème de la gestion ou de l'élimination de cette trame dans le cas d'un composant à haute résolution. Il convient de mentionner des travaux intéressants qui ont permis d'obtenir un fonctionnement bistable dans des cellules épaisses (idem aux nématiques) en mode "ghest host» [25] (utilisation de molécules dichroïques entraînées par le $\mathrm{SmC}^{*}$ ), mais lés contrastes restent faibles.

Une deuxième façon d'améliorer la bistabilité est d'utiliser la «stabilisation par champ alternatif » [26]. On applique un champ alternatif à fréquence suffisamment élevée pour ne pas entraîner les molécules en rotations alternatives entre les deux états adressés. Ce champ tend à rapprocher les molécules d'une position perpendiculaire à lui-même pourvu que l'anisotropie diélectrique du cristal liquide soit négative. L'application d'un champ alternatif en dehors des phases d'écriture et d'effacement sur l'ensemble du matériau modulateur est donc à même de maintenir le contraste des états adressés pendant les phases de mémoire et de lecture [27]. On peut donc utiliser des cellules plus épaisses qui auraient un médiocre 
contraste des états mémorisés si elles. étaient uniquement stabilisées par les surfaces. Il faut noter que-le champ alternatif interagit, avec la polarisation induite du cristal et donc est beaucoup moins efficace que le champ «continu » d'adressage. Cette technique est applicable aux OASLM, máis son efficacité qui dépendra surtout de la technique de photoadressage n'a pas encore été étudiée spécifiquement.

Les deux méthodes précédentes utilisées conjointement donnent des résultats très encourageants dans les applications à adressage électrique matriciel où l'image est naturellement décomposée en pixels aux bords desquels on peut placer les plots d'espacement, de plus les pixels non adressés sont naturellement soumis à un champ alternatif.

Une troisième voie poür améliórer la bistabilité consisterait à utiliser une áutre phase ferroélectrique: la phase I*.'Certains mélanges de cristaux liquides possèdent une phase smectique I* où a été observée une «bistabilité de masse " [23] (où les surfaces jouent un rôle secondaire) qui semble provenir des liaisons tridimensionnelles entre molécules qui existent dans cette phase [28]. Ces mélánges sont évidemment nettement plus visquéux que les SmC* et donc moins rapides. Ils ont donc été beaucoup moins développés que les $\mathrm{SmC}^{*}$. La possibilité de réaliser des cellules raisonnablemént fines et réellement bistables avec ces cristaux semble, a priori, justifier une étude de leur supériorité éventuelle' sur les SmC*'dans les OASLM rapides et bistables.

- Temps d'écriture : pour pouvoir augmenter la vitesse de commutation (5), l'accent a été porté sur l'augmentation de la polarisation spontanée par addition de dopants chiraux plutôt que sur la diminution de la viscosité. En fait ceci ne conduit effectivement à une augmentation de vitesse qu'avec des mélanges d'une parfaite pureté ionique. En effet, l'équipe du LETI a montré récemment [29] que dans le cas de forte polarisátion spontanée du mélange, la cellule commute certes très rapidement dès l'application dù champ de l'état mémorisé ( $M-$ par exemple) vers l'état adressé opposé $(\mathrm{A}+)$ mais recommute à' la même vitesse vers l'état mémorisé précédent $\left(\mathrm{M}_{-}\right)$dès la coupure du champ. Cette recommutation inverse est due à l'existence d'un champ ionique (dû aux inévitables impuretés ionisées du mélange) qui se crée durant la mémorisation et se renforce pendant l'application du champ extérieur. Des temps d'écriture courts sont donc favorisés dans l'ordre décroissant d'importance par: une faible viscosité, une grande pureté ionique (résistivité élevée), une forte polarisation spontanée.

La sensibilité en température du temps d'écriture est élevée : le temps d'écriture est divisé par 2, chaque fois que la température augmente de $6{ }^{\circ} \mathrm{C}$.

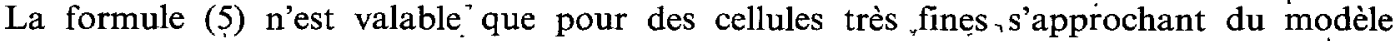
simplifié et dans le cas de champs suffisamment élevés pour négliger les forces d'orientation de surface. Les champs réellement appliqués sont généralement plus faibles. Les temps d'écriture observés (comme ceux des Figs. $6 \mathrm{~b}$, $\mathrm{c}$, d) sont donc en général nettement plus grands que ceux donnés par (5). Des temps d'écriture de $30 \mu$ s sont couramment àtteints sous quelques dizaines de volts par micron (pour satisfaire à la cadence vidéo grand public de $64 \mu$ s/ligne).

- La résolution : elle est en fait limitée par la densité de défauts «zig-zàg ». En effet, deux structures de couches théoriquement équiprobables sont stabilisées par les surfaces figure 4. A la jonction entre ces deux structures locales, des défauts apparaissent qui forment des zigzag ou des lignes allongées lors de l'observation de la cellule au microscope. Heureusement, dans les cellules fines la cohabitation.des deux structures locales de couche n'èst pas énergétiquement favorable, il suffit donc d'une légère dissymétrie (en utilisant par exemple un frottage parallèle des 2 surfaces) dans des couches d'alignement qui doivent d'autre part être parfaitement homogènes pour, qu'une seule structure s'impose sur toute la surface. La densité de défauts devient alors très faible. Une parfaite homogénéité des couches 
d'alignement leụ impose souvent d'être assez épaisses ( $1500 \AA$ ) et d'adhérer parfaitement à une couche homogène sous-jacente. Souvent une couche intermédiaire qui assure une fonction de lissage et d'adhésion est nécessaire $\left(\mathrm{SiO}_{2}\right.$ par exemple). On peut encore diminuer la densité de défauts par leur ancrage entre les plots d'espacement par application d'un champ électrique lors de la descente en température après remplissage.

Il est 'clair que l'on pourrait atteindre des résolutions très bonnes de l'ordre de $100 \mathrm{lp} / \mathrm{mm}$ avec des rapports de contraste de 100 , mais les travaux antérieurs sur les valves optiques ayant montré que la résolution globale du composant était limitée par le photoadressage et non par le cristal. liquide, l'effort doit d'abord être porté sur cet aspect.

\section{Photòadressàge des cristaux liqüides ferroélectriques.}

Les méthodes d'adressage des cristaux liquides à l'aide d'un matériau photoconducteur sont désormais classiques. Elles reposent sur une analyse longitudinale puis transversale.

L'analyse longitưdinale consiste à établir un schéma équivalent de l'empilement de matériaux qui constitụe le composant lorsqu'il est soumis à un éclairage uniforme d'intensité variable. Ce schéma équivalent est généralement constitué de deux impédances: $Z_{\mathrm{PH}}$ celle du photoconducteur, $Z_{\mathrm{CL}}$.celle du cristal liquide en série. Ces impédances sont généralement essentiellement capacitives et seule la composante résistive de $Z_{\mathrm{PH}}$ varie avec l'éclairement [6]. La tension de pilotage est appliquée aux bornes. de ces deux impédançes en série. Ce schéma permet de formuler les contraintes sur la géométrie des matériaux et les caractéristiques de la tension de pilotage pour que le cristal liquide commute sous éclairement et ne commute pas dans l'obscurité. Dans le cas des cristaux liquides nématiques, la tension de pilotage est choisie sinusoïdale, on exprime la valeur efficace de la fraction de cette tension qui apparait aux bornes du cristal liquide en fonction de l'éclairement. Le choix de la fréquence de pilotage rajoute un degré de liberté qui permet d'optimiser la sensibilité en intensité. Le cristal liquide nématique commute lorsque l'intensité lumineuse (qui définit la sensibilité du composant) est suffisante pour que la tension aux bornes du cristal dépasse sa tensión efficace de saturation.

La même analyse appliquée aux cristaux liquides ferroélectriques est beaucoup plus difficile à mener et plus contraignante. En effet, c'est le' comportement transitoire'du schéma équivalent sur des impulsions courtes devant les constantes de temps du photoconducteur qui entre en jeu.' Le caractère ferroélectrique du cristal liquide accroît la complexité de son schéma équivalent. Enfin on doit toujours veiller à maintenir une valeur moyenne de tension nulle aux bornes du cristal (comme pour tous les cristaux liquides).

L'analyse transversale classique repose sur le modèle électrostatique de Roach [30] qui conduit à la bande passante spatiale du composant et à son optimisation éventuelle dans le cas où l'analyse longitudinale a laissé quelques degrés de liberté. Plus exactement ce modèle permet d'exprimer la fonction de transfert de modulation (FTM), du composant en fonction de la fréquence spatiale du faisceau d'écriture. On l'exprime généralement en pourcentage relativement à sa valeur maximale obtenue pour les fréquences spatiales faibles. La résolution spatiale du composant est conventionnellement la fréquence spatiale où la FTM-vaut $50 \%$. Ce modèle montre que la résolution du composant augmente lorsque les épaisseurs de couche de cristal liquide ou de photoconducteur diminuent. Ceci a été vérifié en diminuant l'épaisseur de cristal liquide [31].

Le silicium amorphe hydrogéné a-Si:H est intéressant de ce point de vue en raison de sa très faible distance d'absorption $(0,5 \mu \mathrm{m})$ dans le visible. Il a été utilisé pour la première fois par Ashley et Davis en 87 pour réaliser des couches photoconductrices fines qui conduisirent effectivement à des bonnes résolutions $(\rightarrow-351 \mathrm{p} / \mathrm{mm})$ avec $\mathrm{du}$ cristal liquide nématique. Très récemment un modulateur spatial utilisant du cristal liquide $\mathrm{SmC}^{*}$ (SCE 3 de BDH limited) 
[33] et du a-Si:H comme photoconducteur a conduit à une résolution de $28 \mathrm{lp} / \mathrm{mm}$ (à $50 \%$ de FTM), un temps d'écriture de $250 \mu$ s et une meilleure sensibilité dans le visible que la valveHughes (qui a une résolution comparable).

Toutefois, l'épaisseur des couches de a-Si:H employées $(2 \mu \mathrm{m})$ ne permet pas d'utiliser ce composant en transmission comme décrit au paragraphe 4. D'autre part, la bistabilité du composant n'est pas caractérisée. Bien que des essais avec d'autres matériaux [34] (BSO) aient été tentés, le silicium amorphe hydrogéné semble pourtant s'imposer comme matériau de photoadressage tant par la résolution qu'il permet que par ses avantages technologiques. La technologie silicium $[38,39]$ basse température $\left(200^{\circ} \mathrm{C}\right)$ permet des dépôts d'épaisseur bien contrôlée sur du verre lui-même recouvert d'électrodes transparentes $\left(\mathrm{SnO}_{2}\right.$, ITO...). D'autre part, la possibilité de dopage du silicium amorphe permet d'envisager des dispositifs à jonction (photodiode $\mathrm{p}-\mathrm{i}-\mathrm{n}$ par exemple) qui ouvrent la possibilité d'utiliser d'autres configurations de photoadressage que la photoconduction analysée plus haut. Le silicium amorphe ne peut pas orienter seul des couches de cristal liquide smectique $C^{*}$ de bonne qualité (faible densité de défaut zig-zag). Par conséquent, des couches intermédiaires (silice + polymère frotté) peuvent être nécessaires. Pour le silicium amorphe et en raison de sa conductivité, le modèle électrostatique de Roach ne convient plus.

Compte tenu des difficultés des analyses longitudinales et transversales spécifiques du silicium amorphe et du cristal liquide ferroélectrique, une configuration à photodiode $\mathrm{p}-\mathrm{i}-\mathrm{n}$ a été proposée $[35,42]$ puis expérimentée $[36,41]$. Une configuration transmissive mixte photoconducteur-photodiode à trois électrodes a été expérimentée [37] sur du cristal liquide nématique avec une modélisation matricielle par éléments localisés de l'ensemble du composant dont le principe pourrait être appliqué à la modélisation globale du composant symétrique transmissif que nous proposons.

\section{Etude expérimentale.}

On se propose d'illustrer le principe d'une configuration photodiode tête-bêche par le montage de la figure 6. La cellule SSFLC utilisée au paragraphe 6 (de surface $1 \mathrm{~cm}^{2}$ ) est montée en série avec deux photodiodes p-i-n au silicium amorphe hydrogéné, montées têtebêche, d'une épaisseur de couche intrinsèque de $1 \mu \mathrm{m}$. L'ensemble des trois composants est alimenté par la tension de pilotage $V_{\mathrm{p}}$ synchronisée avec les impulsions lumineuses de puissance $P_{\text {opt }}$ qui éclairent les photodiodes. Un tel ensemble matérialise sous forme éclatée le schéma équivalent d'un seul pixel d'un OASLM constitué d'un empilement de deux couches $\mathrm{p}$-i-n de a-Si:H et d'une couche de SSFLC (Fig. 7). L'unique longueur d'onde (laser $\mathrm{He}-\mathrm{Ne}$ ) d'écriture et de lecture éclaire simultanément les trois composants. Le faisceau issù d'un laser polarisé linéairement est découpé (par un modulateur acousto-optique) en synchronisme avec la tension de pilotage, puis il est séparé par une lame semi-transparente en deux faisceaux (un faisceau d'écriture, un faisceau de lecture). Le faisceau d'écriture est luimême séparé en deux par un prisme de Wollaston qui permet d'équilibrer les puissances incidentes sur les photodiodes dont une peut être masquée par une densité étalonnée. Celle-ci simule l'atténuation que subira le faisceau d'écriture dans le composant intégré lorsqu'il traversera la première couche $\mathrm{p}-\mathrm{i}-\mathrm{n}$ avant d'atteindre la seconde couche. Le faisceau de lecture éclaire le même montage que celui du paragraphe 6 (Fig. 5e). On observe le photocourant $I_{\mathrm{ph}}$ qui donne l'intensité transmise par la cellule SSFLC.

Un tel composant inséré dans une architecture de calcul équivalente à celle de la figure 2 passera successivement à chaque cycle de calcul dans les quatre phases de durées identiques (Fig. 7) : lecture ( $\Delta t_{1}$ et $\left.\Delta t_{5}\right)$ - effacement par un faisceau uniforme $\left(\Delta t_{2}\right.$ et $\left.\Delta t_{6}\right)$ - écriture par un faisceau porteur d'information $\left(\Delta t_{3}\right.$ et $\left.\Delta t_{7}\right)$ - mémorisation $\left(\Delta t_{4}\right.$ et $\left.\Delta t_{8}\right)$. La tension de pilotage périodique (Fig. 7) est fournie par une séquence de deux bi-impulsions symétriques 


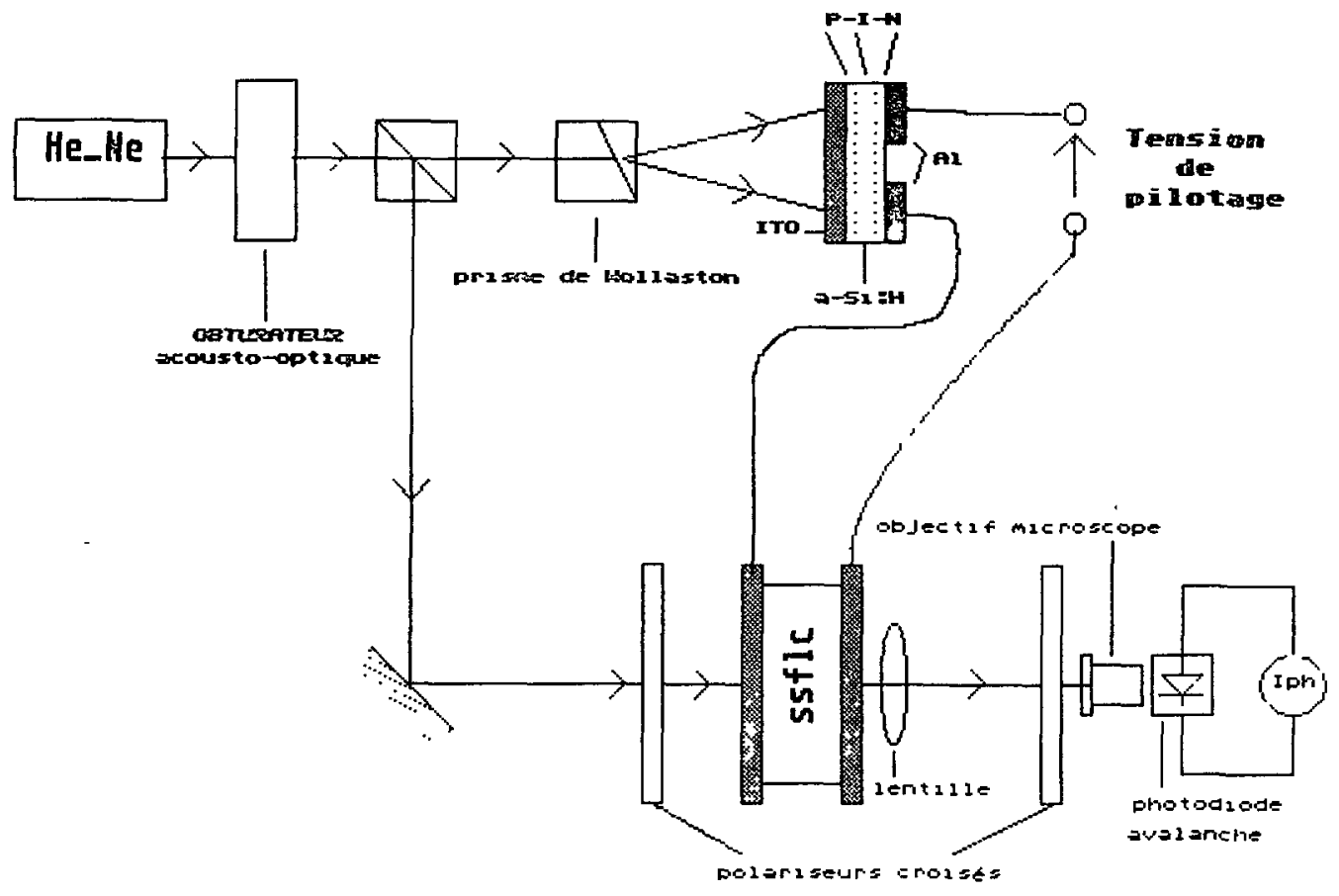

Fig. 6. - Montage utilisé pour commuter une cellule SSFLC optiquement par deux photodiodes a-Si:H montées tête-bêche.

[Experimental set-up performing SSFLC switching optically using two positioned in a mirror image aSi:H photodiodes.]

brèves de séquence donnée (positive puis négative par exemple comme indiqué sur la Fig. 7) pendant la phase d'effacement et de séquence inverse pendant la phase d'écriture. La tension de pilotage est maintenue nulle pendant les phases de lecture et de mémorisation. Dans une architecture de calcul les différentes sources lumineuses pulsées de lecture, d'effacement et d'écriture sont différentes, à telle fin qu'il a été ménagé dans l'expérience des temps d'extinction entre chaque phase de calcul simulant les temps de commutation inter sources d'une configuration réelle en cours de fonctionnement. Les impulsions lumineuses d'effacement et d'écriture sont plus larges et encadrent la bi-impulsion de pilotage correspondante.

On adopte, par exemple, les conventions de logique suivantes : lorsque la cellule a ses axes optiques parallèles aux polariseurs elle sera dite dans un état logique « 1 » qui se traduira par un niveau bas sur la réponse $I_{\mathrm{ph}}$. A l'écriture on prendra le niveau haut de puissance optique comme « 1 " logique. Le montage est soumis à une alternance de deux cycles de calcul. Dans un premier cycle on écrit un « 1 » dans le composant (la phase $\Delta t_{3}$ ), cette valeur mémorisée (pendant $\Delta t_{4}$ ) est ensuite lue dans le cycle suivant (cycle 2 , phase $\Delta t_{5}$ ), après remise à zéro (effacement pendant $\Delta t_{6}$ ) de la cellule, on écrit un « 0 » (absence d'impulsion d'écriture pendant $\Delta t_{7}$ ) qui est mémorisé (pendant $\Delta t_{8}$ ), puis lu (pendant $\Delta t_{1}$ ) au cycle suivant (cycle 1) et effacé (pendant $\Delta t_{2}$ ). Chaque cycle effectue ainsi la lecture du résultat écrit lors du cycle précédent et mémorisé, puis écrit une autre information après effacement. 

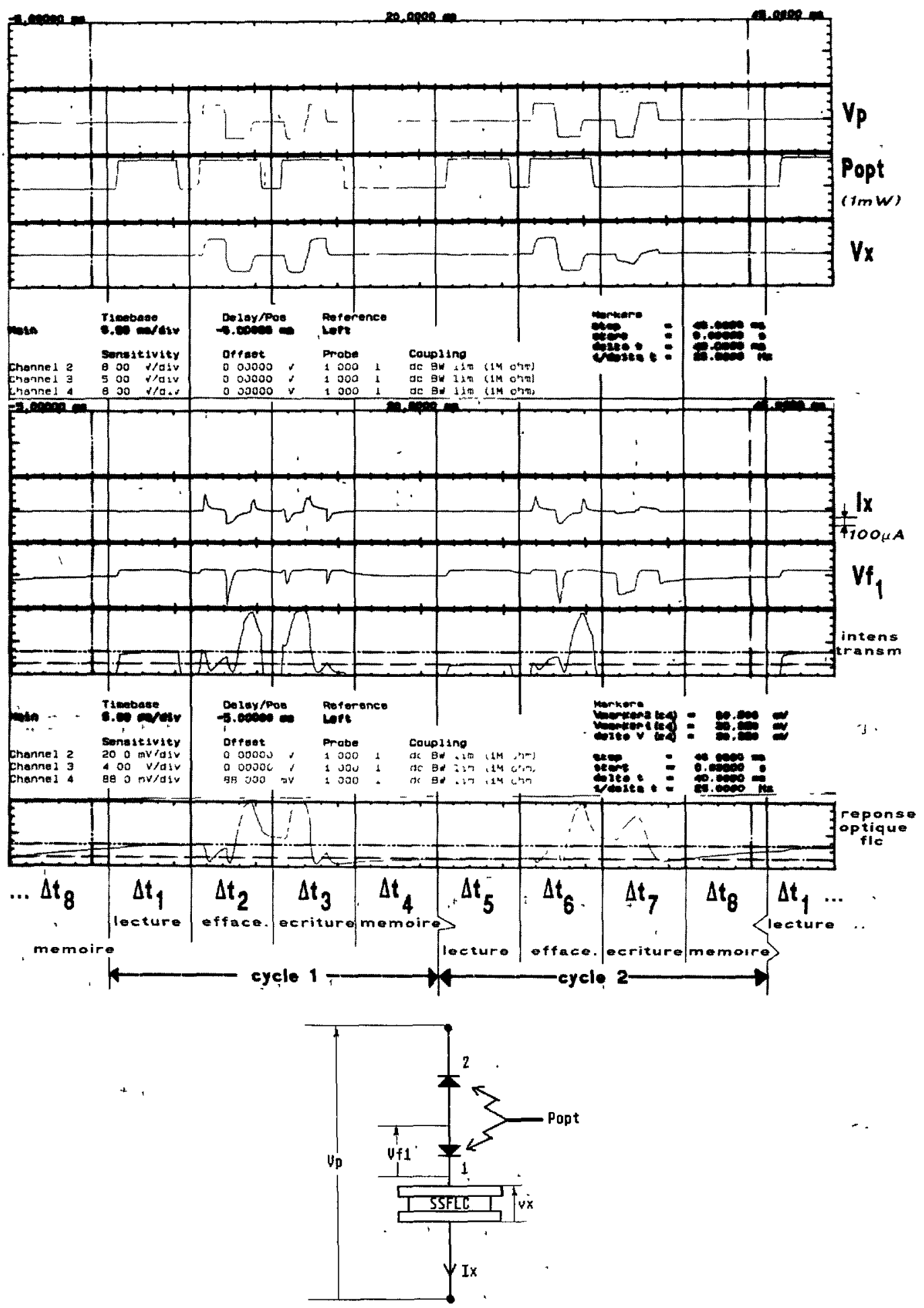

Fig. 7. - Formes d'onde appliquées et observées sur deux cycles de calcul du montage de la figure 6. [Different waveforms occurring in the experimental set-up of figure 6 during two computing cycles.] 


\section{Résultats et analyse.}

- Les résultats : sur la figure 7 apparaissent les évolutions des grandeurs électriques et optiques référencées sur le schéma de la même figure. L'intensité transmise n'est porteuse d'information utile que pendant.les intervalles de lecture $\Delta t_{1}$ et $\Delta t_{5}$. Dans une architecture comme celle représentée en figure 2 , l'intensité transmise à ces instants constitue en effet l'intensité d'écriture du résultat précédent (du SLM1 par exemple) dans l'autre modulateur (SLM2). Il est clair, compte tenu des conventions définies précédemment que, d'une part durant $\Delta t_{5}$ du $2^{\text {eme }}$ cycle on lit l'état « 1 » qui a donc été enregistré correctement durant $\Delta t_{3}$ et qui est resté mémorisé durant $\Delta t_{4}$ et que, d'autre part, on lit durant $\Delta t_{1}$ l'état « 0 » enregistré au cycle précédent durant $\Delta t_{7}$ et mémorisé durant $\Delta t_{8}$. L'intensité transmise est bien sûr découpée comme cela s'observerait normalement dans une architecture du type représenté en figure 2. La réponse optique de la cellule (dernière trace Fig. 7), qui est l'intensité transmise lorsque le faisceau incident n'est pas découpé, permet d'observer en permanence le comportement optique du cristal liquide. Cette réponse montre que durant la phase d'écriture d'un état « $1 »$ dans le cycle $1\left(\Delta t_{3}\right)$ le cristal liquide initialement dans, un état transmissif commute dans un état absorbant sous l'influence de la tension $V_{\mathrm{x}}$ qui apparaît aux bornes du cristal liquide elle-même très proche de la tension de pilotage $V_{\mathrm{p}}$. Par contre, la même réponse observée durant la phase d'écriture de l'état « 0 » du cycle 2 (phase non adressée $\Delta t_{7}$ montre que le cristal liquide dans le même état initial étant soumis cette fois à un résidu de tension $V_{\mathrm{x}}$ fortement atténuée par rapport à la tension de pilotage ne peut commuter et retourne pendant le temps de mémorisation $\Delta t_{8}$ dans le même état.initial. On observe également que les phases d'effacement ramènent toujours, quel que soit l'état initial, vers un état final toujours transmissif. On mesure en utilisant des densités étalonnées un rapport en puissance de $2(3 \mathrm{~dB})$ entre les deux états lus. Une puissance de $0,5 \mathrm{~mW}$ sur chaque diode (de surface $0,3 \mathrm{~cm}^{2}$ environ) est nécessaire pour assurer ce contraste sur $1 \mathrm{~cm}^{2}$ de cristal liquide avec une tension de pilotage appliquée de $\pm 4 \mathrm{~V}$. Chaque phase dure $5 \mathrm{~ms}$ et donc un cycle de calcul dure $20 \mathrm{~ms}$, soit une cadence de 50 cycles de calcul par seconde.

- Analyse d'une phase pilotée et adressée: le fonctionnement observé dans une telle phase $\left(\Delta t_{2}, \Delta t_{3}, \Delta t_{6}\right)$ peut s'analyser de la façon suivante. A l'état initial, la tensión $V_{\mathrm{x}}$ aux bornes du cristal liquide est nulle ainsi que le courant $i_{\mathrm{x}}$ dans le circuit et la tension de pilotage $V_{\mathrm{p}}$. Dans cet état, une impulsion lumineuse n'entraîne aucun courant notable et les deux tensions directes $V_{\mathrm{F} 1}$ et $V_{\mathrm{F} 2}$ sont égales à la tension photovoltaïque en circuit ouvert $V_{\text {oc }}$ (environ $0,7 \mathrm{~V}$ ) de la diode correspondante qui dépend peu de l'éclairement ; ces deux ténsions se compensent mutuellement. Ce dernier fonctionnement est aussi celui des phases de lecture 'qui sont optiquement adressées, mais non pilotées. Si on applique maintenant brutalemerit une tension $V_{\mathrm{p}}$ positive (première partie de la bi-impulsion d'effacement par exemple pendant $\Delta t_{2}$ ), la variation de $V_{\mathrm{p}}$ égale à sa valeur crête se trouve "entièrement reportée en inverse sur la diode 2 qui fournit quasi instantanément (à l'échelle des temps de cycle observés) un photocourant positif qui atteint une valeur crête de $150 \mu \mathrm{A}$. Ce courant est directement proportionnel à la puissance optique totale reçue par la photodiode 1 avec un coefficient de conversion de $S \doteq 0,33 \mathrm{~A} / \mathrm{W}$ qui dépend peu de la tension inverse appliquée comme le montrent les relevés statiques de caractéristiques courant-tension qui ont été effectuées sur, les photodiodes utilisées. Les courbes représentées figure 7 correspondent au cas où la puissance optique incidente $P_{\text {opt }}$ est partagée également (par réglage du prisme de Wollaston) entre les deux photodiodes. Chacune reçoit donc $0,5 \mathrm{~mW}$ ce qui correspond à un courant crête d'environ $150 \mu \mathrm{A}$ comme observé. Ce photocourant circule dans la cellule qui se comporte initialement comme une-capacité pure, la tension $V_{\mathrm{x}}$ à ses bornes croît donc linéairement jusqu'à ce qu'elle vienne compenser exactement la tension de pilotage. Ceci 
entraîne du même coup une décroissance linéaire de la tension inverse de la diode 2 qui repasse en polarisation directe sur le coude photovoltaïque ce qui provoque une décroissance rapide du photocourant. Si la puissance optique est suffisante, la tension aux bornes de la cellule atteint sa valeur maximale en un temps $t_{\mathrm{r}}$ très court devant le temps de commutation du cristal liquide qui peut donc être effectivement assimilé à une capacité $C_{0}$ constante pendant ce temps. Il en résulte :

$$
t_{\mathrm{r}} \approx C_{0} \cdot \hat{V}_{\mathrm{p}} / S \cdot \hat{P}_{\mathrm{opt}}
$$

où $\hat{V}_{\mathrm{p}}$ et $\hat{P}_{\mathrm{opt}}$ sont les valeurs crêtes de $V_{\mathrm{p}}$ et $P_{\text {opt }}$ et $S$ le coefficient de conversion mentionné précédemment. Après cette charge quasi instantanée de la cellule, il s'y établit un champ électrique qui interagit avec la polarisation spontanée des couches de cristal liquide pour provoquer une rotation (que l'on observe par l'intensité transmise) des molécules avec une vitesse qui dépend des caractéristiques physiques du cristal liquide (viscosité, polarisation spontanée), de l'état initial où se trouve le cristal $\left(\mathrm{A}_{+}, \mathrm{A}_{-}, \mathrm{M}_{+}, \mathrm{M}_{-}\right)$et de l'amplitude du champ appliqué. Dans tous les cas, cette rotation moléculaire entraînerait une diminution de la tension aux bornes de la cellule si celle-ci était laissée isolée. Ici, il s'établit par autorégulation sur le coude photovoltaïque de sa caractéristique un équilibre dynamique : la photodiode Dl injectant le photocourant juste nécessaire pour que $V_{\mathrm{x}}$ compense la tension de pilotage au fur et à mesure que le cristal liquide commute. En résumé, chaque impulsion de courant présente quatre parties : temps de montée très rapide qui est lié au temps de montée de la photodiode - palier pendant la durée $t_{\mathrm{r}}$ - décroissance rapide sur le coude photovoltaïque - décroissance quasi exponentielle durant l'équilibre dynamique. Lorsque l'on inverse brutalement la tension de la bi-impulsion de pilotage la variation de deux fois la valeur crête se trouve presque entièrement reportée en inverse sur la diode 1 qui était précédemment polarisée en direct. On retrouve donc le même phénomène qu'au front montant de la bi-impulsion avec échange des diodes jusqu'à ce que $V_{\mathrm{x}}$ compense la tension négative de pilotage. La durée du phénomène est un peu plus longue car on part d'une valeur initiale de $V_{\mathrm{x}}$ légèrement positive et non plus nulle. L'amplitude crête de l'impulsion négative de courant est toujours de $150 \mu \mathrm{A}$ car elle ne dépend que de la puissance optique qui n'a pas changé. On retrouve ensuite les 2 phases de décroissance du courant $i_{x}$ comme déjà mentionné. Enfin, lorsque l'on ramène à zéro la tension de pilotage (fin de la bi-impulsion) les diodes restent d'abord sous éclairement comme on se l'est imposé. La variation brutale de tension de pilotage se retrouve presque entièrement reportée en inverse sur la diode 2 qui fournit alors suivant le processus désormais classique une impulsion de courant positive de $150 \mu \mathrm{A}$ ayant pour rôle de ramener brutalement à zéro la tension $V_{\mathrm{x}}$ aux bornes du cristạl liquide. Viennent ensuite les phénomènes de décroissance du courant qui s'opèrent sur le coude photovoltaïque de la diode 2 , cette fois, pendant lesquels la rotation moléculaire a lieu dans le cristal liquide sous tension maintenue nulle. On supprime la puissance optique lorsque le courant $I_{\mathrm{x}}$ est redevenu nul, la rotation moléculaire est alors terminée et la cellule est dans un état stable mémorisé. Durant une phase adressée chaque diode subit donc soit deux impulsions de tension inverse d'amplitude $V_{\mathrm{p}}$, soit une seule impulsion d'amplitude $2 V_{\mathrm{p}}$ (voir trace $V_{\mathrm{F} 1}$ ).

- Analyse d'une phase pilotée non adressée $\left(\Delta t_{7}\right)$ : dans une telle phase, on observe tout de même une notable sollicitation purement électrique (les diodes étant dans l'obscurité) de la tension de pilotage sur la cellule. L'état initial est toujours $i_{\mathrm{x}}=0, V_{\mathrm{x}}=0$. Une transition négative de la tension de pilotage se trouve partagée entre la tension inverse aux bornes de la diode 1 et aux bornes de la cellule dans un rapport qui peut grossièrement être approximé par le rapport des capacités de la diode en inverse et de la cellule qui sont malheureusement du 
même ordre. Durant la partie négative de la bi-impulsion de pilotage, la fraction de tension aux bornes de la cellule a tendance à se renforcer en raison d'un faible courant inverse d'obscurité de la diode 1 et également par l'existence d'un courant superficiel transversal dans la zone à dopage $n+$ entre les deux cathodes des diodes qui sont en fait prises dans la même couche de a-Si:H.

\section{Discussion.}

Classiquement, l'utilisation de cristal liquide impose qu'en tout point la valeur moyenne du champ électrique appliqué sur un cycle de calcul soit rigoureusement nulle. Dans les cellules à cristal liquide nématique, ceci est facilement vérifié car la commande s'effectue par modulation d'amplitude de tensions sinusoïdales à fréquence relativement élevée devant celle de calcul. Pour les cristaux liquides ferroélectriques la contrainte en question est plus difficile à satisfaire. En effet, l'effet électro-optique prépondérant dans les cristaux liquides ferroélectriques est proportionnel au champ électrique appliqué et non à son carré comme dans les nématiques. La commutation ne peut donc être obtenue par simple variation de la tension efficace de tensions alternatives à valeur moyenne nulle. Dans l'expérimentation réalisée, la symétrie de la configuration assurée par les deux diodes tête-bêche, la symétrie des impulsions de pilotage, leur synchronisation avec les impulsions optiques entraînent la nullité de la valeur moyenne de la tension aux bornes du cristal liquide (trace $V_{\mathrm{x}}$ Fig. 7) sur chaque cycle de calcul quelle que soit la donnée écrite. De plus cette symétrie permet, en inversant le sens de toutes les bi-impulsions de pilotage, d'enregistrer et de mémoriser la même image incidente sous forme d'états $\mathbf{M}-$ (au lieu d'états $\mathbf{M}+$ comme choisi arbitrairement dans l'expérimentation).

Ceci permet de pratiquer une opération d'inversion sur l'image durant un cycle de calcul par inversion électrique de la tension de pilotage. L'utilisation d'une seule photodiode [35] (par exemple en supprimant la diode 1 de la Fig. 7) supprimerait le photoadressage des impulsions positives de pilotage, ce qui impliquerait forcément une valeur moyenne positive aux bornes de la cellule puisque toutes les impulsions positives seraient répercutées alors que les impulsions négatives ne le sont que si le pixel correspondant est adressé. Les impulsions de pilotage de la figure 7 devraient donc être dissymétrisées pour favoriser l'aire des impulsions négatives en raison inverse de la probabilité d'adressage d'un pixel, ce qui suppose que celleci soit connue et fluctue peu avec le temps. Dans certaines applications [9] ceci peut être obtenu à condition d'inclure à chaque cycle de calcul une inversion de l'image en intensité ; la probabilité d'adressage fluctue peu autour de $1 / 2$ et des impulsions négatives d'aire double de celle des impulsions positives permettraient de maintenir une valeur moyenne de tension nulle sur chaque pixel. Evidemment l'absorption d'un composant à une seule couche de aSi:H au lieu des deux qu'impose un montage tête-bêche est bien plus faible. Mais la bistabilité intrinsèque d'un composant de taille raisonnable (quelques $\mathrm{cm}^{2}$ ) à une seule couche n'a pas encore été obtenue de façon reproductible en raison probablement de la dissymétrie des surfaces de stabilisation. Lorsque l'on utilise le montage à deux diodes tête-bêche le composant peut être réalisé de façon totalement symétrique : le cristal liquide se trouvant entre les deux couches de a-Si:H. Ce composant qui n'a pas encore été réalisé présenterait vraisemblablement une meilleure bistabilité intrinsèque puisque les deux surfaces de stabilisation seraient les mêmes.

Les résultats présentés figure 7 montrent une cadence de calcul très modeste qui ne met en valeur ni les cristaux liquides ferroélectriques, ni l'adressage par photodiode. Ceci est lié d'abord à l'utilisation de cristaux liquides dont la polarisation spontanée est volontairement limitée afin de garantir la bistabilité conformément aux travaux du LETI déjà mentionnés relatifs aux effets du champ ionique. Ces cristaux liquides ont été optimisés pour qu'utilisés 
sous plusieurs dizaines de volts, ils puissent atteindre les cadences vidéo avec un fort taux de multiplexage. Or dans l'expérimentation la tension appliquée (de l'ordre de $4 \mathrm{~V}$ ) est limitée d'une part pour satisfaire à la tenue en tension inverse des photodiodes utilisées (de l'ordre de $6 \mathrm{~V}$ ) et d'autre part pour éviter que le résidu de tension appliqué au cristal liquide par effet capacitif lors de phases pilotées et non adressées n'entraîne une fausse commutation. L'augmentation de vitesse passe donc d'une part par l'optimisation de la tenue en tension inverse des photodiodes tout en gardant une épaisseur faible et une résistivité superficielle élevée. Ceci peut amener à graver la couche dopée en contact avec le cristal liquide et donc à " pixeliser » le compósant. Des ténues en tension de quelques dizaines de volts semblent raisonnables. D'autre part il faut utiliser des cristaux liquides ferroélectriques à forte polarisation spontanée et des cellules plus épaisses pour diminuer le couplage capacitif dans les phases non adressées. Ces deux points contribueront conjointement à une diminution de la bistabilité intrinsèque qui devra donc être assistée par une stabilisation par champ alternatif pour que le composant devenu rapide puisse garder ses propriétés fondamentàles de régénération et de mémorisation.

L'augmentation de puissance optique par rapport à celle mentionnée dans le paragraphe 10 n'apporte que peu de changements quant à là cadence de calcul et au contraste des états mémorisés. En effet cette puissance ne joue que sur le temps $t_{\mathrm{r}}$ (estimé plus haut) qui est petit devant le temps de commutation du cristal liquide. Donc au-delà de cette puissance une forte dissymétrie de puissance optique reçue entre les deux photodiodes n'entraîne que peu de changement dans la réponse optique du composant:

Pour l'utilisation du composant à deux couches dans une architecture de calcul du type présenté plus haut, 'cette dissymétrie doit être prise en compte car la couche arrièré ne recevra que la lumière transmise par la couche avant. La densité de puissance optique très modeste nécessaire dans l'expérimentation résulte de la relative lenteur du cristal liquide mais on sait [35] que l'utilisation de cristaux plus rapides nécessiterait des densités de puissance assez importantes. L'adressage par photodiode est en effet beaucoup moins sensible que l'adressage photoconducteur puisqüe l'on ne bénéficie plus du gain de photoconduction. Cette faible sensibilité n'est pas un handicap majeur dans les applications de calcul optique où l'on est maître de la puissance des sources.

Les seules sources pratiquement utilisables dans une application réelle seraient en effet dẹs diodes laser de puissance qui émettent aux alentours de $830 \mathrm{~nm}$. A cette longueur d'onde la couche de silicium amorphe sera quasiment transparente et n'aura plus aucune efficacité. On peut alors utiliser un alliage de $\mathrm{a}-\mathrm{Si}_{(1-x)} \mathrm{Ge}_{x}$ [43] dans la couche intrinsèque des photodiodes et ajuster le paramètre $x$ pour assurer le compromis efficacité-transparence à $830 \mathrm{~nm}$. Une valeur de $x=0,82$ donne [44] un coefficient à absorption proche de $10^{4} \mathrm{~cm}^{-1}$ pour $830 \mathrm{~nm}$. Un tel matériau a été utilisé [46] dans un empilement de 3 photodiodes ITO/n-i-p/n-i-p/n-i$\mathrm{p} /$ métal - pour la couche intrinsèque de la diode côté métal. Des travaux récents [45] permettent d'envisager l'utilisation de structure amplificatrice à avalanche en utilisant une alternance de couches a-Si:H et $\mathrm{a}-\mathrm{SiC}: \mathrm{H}$ dans la zone intrinsèque, des gains optiques de 180 aux longueurs d'ondes de l'hélium néon sous des tensions inverses de $20 \mathrm{~V}$ et des temps de commutation de $4,5 \mu$ s ont été mesurés. Un tel dispositif permettrait de travailler avec les diodes laser visibles actuellement disponibles. De telles structures ont des épaisseurs du même ordre que celles des photodiodes amorphes classiques et devraient être moins absorbantes compte tenu du gap plus élevé du a-SiC:H devant celui du a-Si:H.

Réalisé en dissymétrique, avec une 'seule' couche photodiode ou à deux couches empilées l'une sur-1'autre, le composant ést toujours utilisable en réflexion. Dans ce cas il est important de noter que les impulsions optiques de lecture (de même longueur d'onde que celles d'écriture) intervenant dans des phases non pilotées n'ont paś besoin d'être optiquement isolées des couches de photoadressage. L'utilisation des couches de blocage habituelles que 
l'on rencontre dans les valves optiques nématiques n'est pas nécessaire. La réflexion du faisceau de lecture peut se faire simplement sur la discontinuité d'indice à l'interface des couches de photoadressage et $\mathrm{du}$ cristal liquide. Celle-ci peut se faire avec un angle d'incidence assez grand compte tenu de l'angle de vue des cellules ferroélectriques plus grand que celui des nématiques.

Enfin il est important de noter la grande sensibilité à la température du contraste des états lus dans l'expérimentation. Une augmentation de température de quelques degrés de la cellule a permis d'atteindre des contrastes de $8 \mathrm{~dB}$ (et non de $3 \mathrm{~dB}$ ). Ceci est une conséquence de la sensibilité à la température de la stabilisation par les surfaces. Cette sensibilité à la température intervient dans une architecture de calcul binaire comme une réduction de l'immunité au bruit.

Dans le cadre des travaux actuellement amorcés sur des composants de taille opérationnelle fonctionnant à cadence maximale, la puissance électrique et lumineuse absorbée sera évaluée ainsi que le comportement thermique du composant seul, de façon' à définir des dispositifs additionnels de refroidissement éventuellement régulés pour maintenir le cristal liquide dans une bande étroite de température, à l'intérieur de la plage de température de sa phase smectique $\mathrm{C}$, où les conditions de régénération et de stabilité de l'architecture considérée sont toujours remplies. La dissipation de puissance peut donc pratiquement intervenir comme un facteur de limitation de la vitesse, d'autant. plus que les marges de fonctionnement de l'architecture sont étroites.

\section{Conclusions.}

Un grand nombre d'architectures de calcul optique utilisent des modulateurs spatiaux de lumière binaires. On analyse les, principales contraintes généralement imposées à ces composants par ces architectures et on constate qu'il n'existe pas actuellement de dispositifs qui les satisfont complètement. On décrit les cristaux liquides ferroélectriques en cellule fine qui, tout en gardant les grandes résolutions des cristaux liquides en général, possèdent en plus une grande vitesse et une possibilité de mémorisation qui conviennent bien pour le calcul optique. On présente les résultats d'essais de telles cellules pour illustrer l'écart du comportement réel par rapport au modèle simplifié et les directions d'optimisation de ces cellules et de leur commande pour cette application. Une méthode de photoadressage originale doit être adaptée à ces cristaux liquides rapides pour en utiliser au mieux les propriétés. Une telle méthode utilisant deux photodiodes au silicium amorphe hydrogéné montées tête-bêche avec des séquences de pilotage optique et électrique adaptées au calcul optique est expérimentée. L'analyse permet d'en déduire l'insuffisance de la seule stabilisation par les surfaces de la cellule à cristal liquide. Ces premiers travaux permettent, plus généralement, de mettre en évidence l'intérêt potentiel de l'association d'un dispositif à base de semiconducteur amorphe en couches minces et d'un cristal liquide binaire, rapide et bistable pour assurer les' fonctions de mémorisation, régénération, amplification et contrôlè qui sont nécessaires à la réalisation d'architectures optiques de calcul binaire.

\section{Remerciements.}

Nous tenons à remercier le LETI pour nous avoir fourni les cellules SSFLC, le laboratoire PICM de l'Ecole Polytechnique pour les photodiodes, ainsi que J.R. Brocklehurst, N. Collings, W. A. Crossland, A. B. Davey (STC Harlow), J. Dijon, T. Leroux (LETI-IRDI), J. P. Le Pesant (Thomson LCR), K. M. Johnson (Université du Colorado-Boulder), J. Equer (PICM), C. Bonnaud (Université de Rennes) pour les discussions fructueuses concernant ces travaux, ainsi que la DRET (Ministère de la Défense) pour son aide financière. 


\section{Bibliographie}

[1] Optical artificial intelligence, numéro spécial Appl. Opt. 26 (1987).

[2] Lohmann A. W. and Weigelt J., Spatial filtering logic based on polarization, Appl. Opt. 26 (1987) 131-135.

[3] Tsvetkov V. A., Morozov N. A. and Elinson M. I., Picture logic and liquid crystals, Sov, J. quant. Electron. 4 (1975) 889-993.

[4] Grinberg J., Jacobson A., Bleha W., Miller L., Fras F., Boswell D. and Myer G., A new real time non coherent to coherent light image converter : the hybrid field effect liquid-crystal light valve, Opt. Eng. 14 (1975) 217.

[5] Efron U., Grinberg J., Braatz P. O., Little M. J., Reif P. G. and Schwartz R. N., The silicon liquid-crystal light valve, J. Appl. Phys. 57 (1985) 1356-1368.

[6] Aubourg P., Huignard J. P., Hareng M. and Mullen R. A., Liquid crystal light valve using bulk monocrystalline BSO as the photoconductive material, Appl. Opt. 21 (1982) 3706-3712.

[7] TANIDA J. and ICHIOKA Y., OPALS : optical parallel array logic system, Appl. Opt. 25 (1986) 1565-1570.

[8] Sawchuk A. A., Strand T. C., Digital optical computing, Proc. IEEE 72 (1984) 758-777.

[9] Cambon P., De Bougrenet de la Tocnaye J. L., Principle of a mathematical morphology processor using ferroelectric liquid crystal light valves, Appl. Opt. 28 (1989) 3456-3461.

[10] Samuelson J., Wieder H., Guarnieri C. R., Chevalier J., Onton A., Fast photoconductor coupled liquid-crystal light valve, Appl. Phys. Lett. 34 (1979) 450-452.

[11] ShamiR J., CAUlfield H. J., Improved architecture for massive holographic interconnection network, Proc. SPIE on Optical Computing (Septembre 1988) pp. 283-287.

[12] Oudar J. L., De nouveaux dispositifs pour la commutation et la logique optique, L'Echo des recherches (CNET) n 134 (1988) pp. 35-42.

[13] Miller D. A. B., Quantum wells for optical information processing, Opt. Eng. 26 (1987) 368-372.

[14] Lagerwall S. T., Wahl J., Clark N. A., Ferroelectric liquid crystal for displays, International display research conference, conference record (San Diego 1985) pp. 213-221.

[15] Dijon J., Ebel C., Maltese P., A 6-in.-Diagonal black and white ferroelectric panel, Proc. of the SID 29/3 (1988) 249-252.

[16] Carlson A., Solar cells, Chapter 2, Semiconductors and Semimetals, part D 21 (1984) pp. 7-35.

[17] Magarino J., Chartier E., Proust N., Szydlo N., Hepp B., Graciet M. et Mourey B., Ecrans à cristal liquide 2. Matrices actives en silicium amorphe, Rev. Tech. Thomson-CSF 18 (1986) 797-844.

[18] Meyer R. B., Liebert L., Strelecki L. and Keller P., Ferroelectric liquid crystals, J. Phys. Lett. France 36 (1975) L-69-71.

[19] Clark N. A., Handschy M. A. and Lagerwall S. T., Ferroelectric liquid crystal electro-optics using the surface stabilized structure, Mol. Cryst. Liq. Cryst. 94 (1983) 213-234.

[20] Rieker T. P., Clark N. A., Smith G. S., Parmar D. S., Sirota E. B. and Safinaya C. R., «Chevron » local layer structure in surface stabilized ferroelectric smectic-C cells, Phys. Rev. Lett. 59 (1987) 2658-2661.

[21] Ebel C., Etude des cristaux liquides smectiques $C$ chiraux en vue de la réalisation d'écrans plats, Thèse d'Etat université Grenoble I (Juillet 1988).

[22] Mulatier L., Etude de la polarité des surfaces d'orientation des cristaux liquides, mémoire CNAM électrochimie Grenoble (Mai 1988).

[23] Crossland W. A., Bone M. and Ross P. W., Prospects and problems of ferroelectric LCDS, SID Eurodisplay (1987).

[24] Ross P. W., $720 \times 400$ matrix ferroelectric display operating at video frame rate, Conf. Digest Int. Display Research conference (San Diego, October 1988).

[25] Isogai M., Kondo K., Kitamura T., MuKoh A., Nagae Y. and KawaKami H., Bistable liquid crystal display device using conventional thickness cell, Jpn Display 86 (1986) pp. 472-474.

[26] Le Pesant J. P., Perbet J. N., Mourey B., Hareng M., Decobert G., Dubois J. C., Optical switching of chiral smectic C at room temperature, Mol. Cryst. Liq. Cryst. 129 (1985) 61-74. 
[27] Umeda T., Nagata T., Mukoh A. and Horı Y., Influences of alignement materials and LC layer thickness on AC field-stabilization phenomena of ferroelectric liquid crystal, Jpn. J. Appl. Phys. 27 (1988) 1115-1121.

[28] Moussa F., Benattar J. J. and Williams C., Positional order and bond orientational order in the liquid crystal smectic F phase, Mol. Cryst. Liq. Cryst. 99 (1983) 145-154.

[29] Dijon J., Ebel C., Vauchier C., Baume F., Clerc J. F., Estor M., Leroux T., Maltese P., Mulatier L., High speed Sc* mixtures : an evaluation of their performances, 1988 SID symposium digest (Anaheim, May 88) p. 246.

[30] ROACH W. R., Resolution of electro optic light valves, IEEE Trans. Electron Devices ED-21 (1974) 453-459.

[31] Baillie W. L., Openshaw P. M., Hart A. D., Makh S. S., Development of liquid crystal light valves using BSO as the photoconductor. Part 2: device performance, Proc. IEEE 133 (Février 1986) 65-69.

[32] Ashley P. R. and DAvis J. H., Amorphous silicon photoconductor in a liquid crystal spatial light modulator, Appl. Opt. 26 (1987) 241-246.

[33] Williams D., Latham S. G., Powles C. M. J., Powell M. A., Chittick R. C., Sparks A. P. and Collings N., An amorphous silicon/chiral smectic spatial light modulator, J. Phys. $D$ : Appl. Phys. 21 (1988) 156-159.

[34] Armitage D., Thackara J. I., Clark N. A. and Handschy M. A., Ferroelectric liquid-crystal and fast nematic spatial light modulator, SPIE, Liquid crystals and spatial light modulateur materials, 684 (1986).

[35] Moddel G., Johnson K. M., Handschy M. A., Photoadressing of high speed liquid crystal spatial light modulators, SPIE, Optical and digital pattern recognition 754 (1987) 207-213.

[36] LI W., KUO C. T., MOdDEl G., JoHNSON K. M., Design and performance of high-speed optically adressed spatial light modulator, SPIE 936; Advances in Optical Information Processing 3 (1988).

[37] Ashley P. R., DAvis J. H. and OH T. K., Liquid crystal spatial light modulator with a transmissive amorphous silicon photoconductor, Appl. Opt. 27 (1988) 1797-1802.

[38] Bisaro R., Chartier E., Kaplan D., Magarino J., Proust N. et Szydlo N., Silicium amorphe préparé par décomposition thermique de silane: propriétés et applications, Rev. Tech. Thomson-CSF 15 (1983) 321-355.

[39] Hydrogenated amorphous silicon part A: preparation and structure, Semiconductors and Semimetals 21 (1984).

[40] Collings N., Crossland W. A., Chittick R. C. and Bones M. F., The novel application of the electroclinic effect to light valve technology, SPIE on Optical Computing (1988) pp. 46-47.

[41] LI W., Kuo C. T. and Moddel G., High speed optically-addressed spatial light modulator, IEEE Trans. Electron Devices 35 (1988) 2448.

[42] Johnson K. and Moddel G., Motivations for using ferroelectric liquid crystal spatial light modulators in neurocomputing, Appl. Opt. 28 (1989) 4888-4889.

[43] Carlson D. E., Solar cells : semiconductors and semimetals, 21, part D, Chapter 2 (1984).

[44] VON ROERDEN B. et al., Optical absorption, photoconductivity and photoluminescence of glowdischarge amorphous a-Si $\mathrm{S}_{(1-x)} \mathrm{Ge}_{x}$ alloys, Phys. Rev. B 25 (1982) 7678-7686.

[45] Jwo Shin-Cherng et al., Amorphous silicon/silicon carbide supperlattice avalanche photodiodes, IEEE Trans. Electron Devices 35 (1988) 1279-1282.

[46] Nakamura et al., 4th European Community Photovoltaic Solar Energy Conference, Stressa, Italy, Ed. D. Reidel (Dordrecht, Holland) 1982, p. 616.

[47] Special Issue on Optical Interconnections, Appl. Opt. 29 (March 1990) 1049-1232.

[48] LeNTINE A. L. et al., Optical logic using electrically connected quantum well PIN diode modulators and detectors, Appl. Opt. 29 (1990) 2153-2163.

[49] PRISE M. E. et al., Module for optical logic circuits using symmetric self-electrooptic effect devices, Appl. Opt. 29 (1990) 2164-2170. 